\title{
炔烃偶联反应研究的新进展
}

\author{
白东虎 ${ }^{a}$ 李春举 ${ }^{a}$ 李 健 ${ }^{a}$ 贾学顺*,,$b$ \\ $\left({ }^{a}\right.$ 上海大学化学系 上海 200444) \\ ${ }^{b}$ 中国科学院上海有机化学研究所 天然产物有机合成化学重点实验室 上海 200032)
}

摘要 综述了近几年来炔偶联反应的新进展, 主要涉及铜试剂和钯试剂催化的端炔偶联反应以及反应机理和应用.

关键词 炔; 炔偶联; 铜催化; 钯催化; 反应

\section{New Progress of Acetylene-Coupling Reactions}

\author{
Bai, Donghu ${ }^{a}$ \\ Li, Chunju $^{a}$ \\ Li, $\operatorname{Jian}^{a}$ \\ Jia, Xueshun ${ }^{*, a, b}$ \\ $\left({ }^{a}\right.$ Department of Chemistry, Shanghai University, Shanghai 200444) \\ ( ${ }^{b}$ Key Laboratory of Synthetic Chemistry of Natural Substances, Shanghai Institute of Organic Chemistry, \\ Chinese Academy of Sciences, Shanghai 200032)
}

\begin{abstract}
The recent studies of acetylene-coupling reactions are reviewed, mainly focusing on copper reagents and palladium reagents catalyzed coupling reactions of terminal alkyne as well as the reaction mechanism and application.

Keywords alkyne; alkyne coupling; copper catalysis; palladium catalysis; reaction
\end{abstract}

端炔的均相偶联反应最早是于 1869 年 Glaser ${ }^{[1]}$ 发现 的, 因此这类反应被称为 Glaser 偶联反应, 端炔偶联反 应作为一种历史悠久的形成 $\mathrm{C}_{\mathrm{sp}}-\mathrm{C}_{\mathrm{sp}}$ 键的方法被人们所 熟知. 目前为止, 国内外用于实现端炔偶联的方法主要 还是基于传统的 Glaser-Hay 端炔氧化均相偶联反应和 Cadiot-Chodkiewicz 交叉偶联反应的继承和发展. 其中 铜试剂催化该类反应受到人们的广泛关注. 近年来钯试 剂催化的炔偶联反应也得到了长足的发展, 此外还有其 它一些促进炔偶联的新方法涌现出来. 该类反应生成的 1,3-共轭二炔类化合物具有刚性结构单元和独特的电子 特性, 这类化合物结构常见于天然产物和抗菌药物中 ${ }^{[2]}$, 同时它还是功能分子材料合成中的重要官能团. 此外炔 偶联反应也在许多合成化学领域得到了广泛的应用, 例 如: 合成多种类型炔化物、构建大环系炔化物, 天然产 物合成和超分子领域的应用等 ${ }^{[4 a]}$. 近年来, 已有综述性 论文对各种类型的炔偶联反应进行了专门的评价 ${ }^{[4,46,5]}$. 鉴于此, 本文从以下几个方面对 2006 2011 年炔烃偶 联反应的研究进展做简要评述.

\section{1 端炔偶联反应}

\section{1 铜试剂催化的端炔偶联反应}

Glaser $^{[1 \mathrm{la}]}$ 于 1869 年首次以苯乙炔为原料, 以氨水和 乙醇为溶剂, $\mathrm{CuCl}$ 为催化剂, 在空气存在条件下成功的 合成了 1,4-二苯基-1,3-丁二炔(Scheme 1). 该反应为非 均相反应, 所以速度较慢, 而且需要分离中间产物苯基 炔化铜, 但由于其结晶性差导致分离困难, 且在干燥条 件下易爆炸出现危险，使得该反应没有得到广泛的应 用 ${ }^{[5]}$.

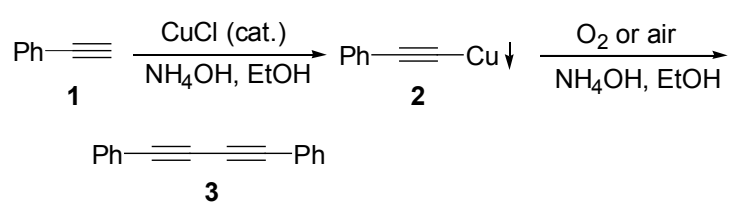

\section{Scheme 1}

另一里程碑式的发展是 1956 年的 EglintonGalbraith 炔烃偶联反应 ${ }^{[6]}$. Eglinton 等 ${ }^{[6]}$ 发现 $\mathrm{Cu}(\mathrm{OAc})_{2}$ 在稀的甲醇一吡啶溶液中可以有效促进端炔偶联反应 (Eq. 1). 因为这一反应为均相反应, 所以反应速度有了

\footnotetext{
*E-mail: xsjia@mail.shu.edu.cn

Received February 7, 2012; revised March 26, 2012; published online April 10, 2012.

Project supported by the National Natural Science Foundation of China (Nos. 21142012, 21002061, 20902057), and the Key Laboratory of Synthetic Chemistry of Natural Substances, Chinese Academy of Sciences.

国家自然科学基金(Nos. 21142012, 21002061, 20902057)和中国科学院上海有机化学研究所天然产物有机合成化学重点实验室资助项目.
} 
很明显的提高. 目前这种方法已经广泛应用于大环系炔 化物的构建中 ${ }^{[7]}$.
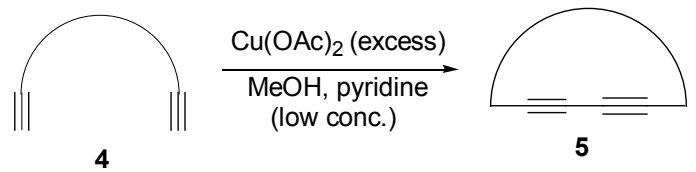

之后, $\mathrm{Hay}^{[8]}$ 对该类反应做了重要改进, 他使用 $\mathrm{CuCl}$ 和 TMEDA 为催化剂在丙酮等溶剂中, 成功地用氧 气氧化端炔合成了二炔类化合物(Eq. 2). 增强反应体系 中活性物种的溶解性是这类反应的一大亮点, 此条件下 的反应又被称为 Hay 偶联反应.

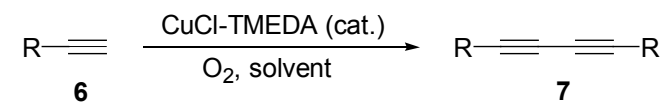

近些年, 国内外报道了许多铜试剂催化的端炔偶联 方法, 多是在以上三种早期经典的氧化偶联基础上的改 进和创新. 已有综述性文章也对这类反应做过总 结 ${ }^{[4 a, 4 b]}$. 因此, 我们仅对最近几年的一些新方法进行了 综述.

Yadav 等 ${ }^{[9]}$ 发现在离子液体 $\mathbf{8}$ 中, Hay 条件下的 Glaser 偶联反应能够顺利进行，该反应的产率可达 $95 \%$, 并且底物炔中官能团对反应影响并不明显(Eq. 3). 随后, Ranu 等 ${ }^{[10]}$ 再次把离子液体 9 应用到该类炔偶联反应中, 并且取得了不错的结果(Eq. 4). 离子液体是常温下呈液 态的离子化合物, 可以溶解多种有机物和无机物. 此类 反应中离子液体同时扮演溶剂和碱的双重作用. 2007 年, Shreere 等 ${ }^{[11]}$ 报道的一例钯催化的端炔偶联反应同样 用到了类似的碱性离子液体. 与一般有机溶剂相比, 离 子液体显现出更好的溶解性和官能团耐受性, 因此可以 更好的加快反应速率, 并且能够有效提高反应产率, 此 外它还可以循环多次催化反应.

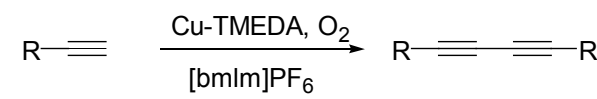

$$
\begin{aligned}
& \frac{{ }_{[\mathrm{bmlm}] \mathrm{PF}_{6}}}{8} \\
& \mathrm{R} \rightleftharpoons \frac{\mathrm{Cul}, \mathrm{O}_{2}}{[\mathrm{bmlm}] \mathrm{OH}} \quad \mathrm{R}=\underset{85 \% \sim 90 \%}{=} \mathrm{R} \\
& \mathrm{OH}^{-} \\
& 9 \\
& \mathrm{R}=\mathrm{NC}\left(\mathrm{CH}_{2}\right), \mathrm{THPO}\left(\mathrm{CH}_{2}\right), \mathrm{TsOCH}_{2}
\end{aligned}
$$

Jiang 研究组 ${ }^{[12 \mathrm{a}]}$ 报道了在超临界二氧化碳中进行的 Glaser 偶联反应, 他利用 $\mathrm{CuCl}_{2}$ 作为氧化剂, 以醋酸钠
代替胺成功的实现了 Glaser 类偶联反应. 在此基础上, 2006 年，该小组又报道了类似的工作 ${ }^{[12 b]}$ ，他们进一步 改进了该反应，提高了收率. 以无机碱 $\mathrm{NaOAc}$ 代替有机 碱既起到碱的作用又作为配体(Eq. 5). 由于 $\mathrm{CuCl}_{2}$ 和 $\mathrm{NaOAc}$ 在 $\mathrm{ScCO}_{2}$ 中的低溶解性, 他们引入甲醇作为助 溶剂, 研究发现, 两种溶剂的比例影响较大, 但 $\mathrm{ScCO}_{2}$ 的温度和压力对反应影响并不大.

$$
\mathrm{R} \equiv \frac{\mathrm{CuCl}_{2}, \mathrm{NaOAc}}{\mathrm{ScCO}_{2}, \mathrm{MeOH}} \mathrm{R}=\mathrm{R}
$$

2009 年, Kuhn 等 ${ }^{[13 a]}$ 发现 Cu-沸石尤其是 Cu(I)-USY 体系能够有效地催化端炔的偶联反应(Eq. 6). 这种非均 相催化剂提供了一种简单方便的无需额外添加碱和配 体的合成 1,3-二炔化物的方法. 实验发现 ${ }^{[13 b]}$ ，沸石气孔 大小对反应影响较大. 此类方法底物范围广，包括芳 基、烷基以及含糖骨架化合物在内的一系列取代炔烃皆 可顺利进行．而且反应过程简洁，催化剂制备方便，易 于回收利用. 之后在 2010 年, Pale 等 ${ }^{[14]}$ 为此类反应提出 了假设的反应机理(Scheme 2). 他们认为 $\mathrm{Cu}(\mathrm{I})$-沸石与 底物炔先形成 $\pi$-络合物中间体 $\mathbf{1 0}$, 然后在氧气条件下, 该中间体重组成炔铜化物. 当两个炔铜化物同处于一个 沸石空腔里时，他们便可形成二炔铜配合物，最后两个 炔化物偶联生成产物. 因此只有具有一定气孔大小的沸 石才可以产生催化效果.

$$
\begin{array}{r}
\mathrm{R}=\frac{\mathrm{Cu}(\mathrm{l})-\mathrm{USY} 30 \%}{\mathrm{DMF}, 110^{\circ} \mathrm{C}} \quad \mathrm{R}=\mathrm{R} \\
\mathrm{R}=\text { Alkyl, Aryl } \quad 80 \% \sim 98 \%
\end{array}
$$

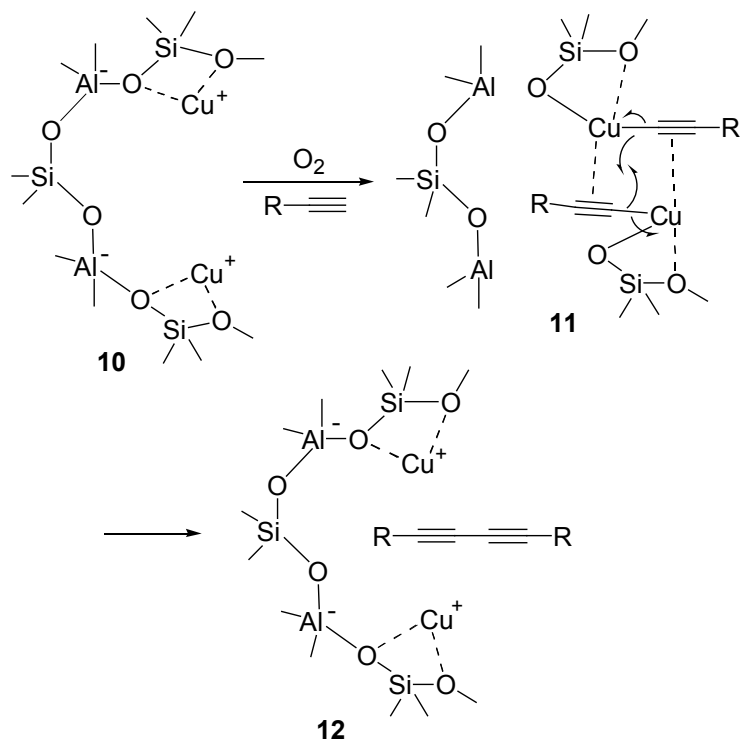

Scheme 2

2010 年, Chen 研究组 ${ }^{[15]}$ 报道了一例无溶剂端炔偶 
联反应. 他们发现以空气作为氧化剂时, 底物端炔与催 化量的 $\mathrm{CuCl}_{2}$ 和三乙胺共热至 $60{ }^{\circ} \mathrm{C}$ 可以成功地合成对 称二炔类化合物. 该反应底物适用性广, 值得一提的是, 该方法既适用于对称二炔的合成，也能够用于合成非对 称的二炔化物(Eq. 7), 只是对于非对称二炔产率尚低. 此外, 这种催化剂循环 5 次使用后仍能保持较高的催化 活性.

$$
\begin{aligned}
& \mathrm{R}^{1}=+\equiv \mathrm{R}^{2} \stackrel{\begin{array}{c}
\mathrm{CuCl}_{2}(3 \mathrm{~mol} \%) \\
\mathrm{Et}_{3} \mathrm{~N}(3 \mathrm{~mol} \%)
\end{array}}{\text { Solvent-free }} \quad \mathrm{R}^{1} \equiv \mathrm{R}^{2} \quad \text { (7) } \\
& 60^{\circ} \mathrm{C} \text {, air } \\
& \mathrm{R}^{1}=\text { Aryl } \mathrm{R}^{2}=\text { Aryl } \quad 55 \% \sim 72 \% \\
& \mathrm{R}^{1}=\text { Alkyl } \mathrm{R}^{2}=\text { Aryl } \quad 32 \% \sim 35 \%
\end{aligned}
$$

最近几年, 我们课题组也发展了一系列铜试剂促进 的端炔偶联方法. 2008 年, 我们发现各类端炔化合物在 等量的 $\mathrm{CuI}$ 促进下, 以碘作添加剂, 碳酸钠为碱的条件 下可以高收率的得到 1,3-二炔类化合物(Eq. 8) ${ }^{[16 \mathrm{c}]}$. 而且 脂肪族的炔也表现出很好的反应性. 随后我们对此反应 进行了改进 ${ }^{[16 b]}$, 发现将 $\mathrm{CuI}$ 的量降至催化量时, 以醋酸 钠为碱、空气作为氧化剂的条件下, 仍然可以顺利完成 此类 Glaser 偶联反应(Eq. 9).

$$
\begin{aligned}
& \mathrm{R} \equiv \frac{\mathrm{Cul}_{2} \mathrm{I}_{2}}{\mathrm{NaCO}_{3}, \mathrm{DMF}} \mathrm{R}=\mathrm{R} \\
& \mathrm{R}=\text { Alkyl } 70 \% \sim 89 \% ; \mathrm{R}=\text { Aryl } 92 \% \sim 99 \% \\
& \mathrm{R} \equiv \frac{5 \mathrm{~mol} \% \mathrm{Cul}, \mathrm{NaOAc}}{\mathrm{DMF}, \text { air, } 90^{\circ} \mathrm{C}} \mathrm{R}=\mathrm{R} \\
& \mathrm{R}=\text { Alkyl } 73 \% \sim 85 \% ; \mathrm{R}=\text { Aryl } 91 \% \sim 97 \%
\end{aligned}
$$

最近, 我们又发现一种操作简便的端炔偶联方法. 这种方法无需配体、碱和其他特殊添加试剂，只需 $\mathrm{CuCl}^{[16 \mathrm{a}]}$ 或 $\mathrm{Cu}(\mathrm{OAc})_{2}\left(\mathrm{H}_{2} \mathrm{O}\right)^{[16 \mathrm{~d}]}$ 促进、空气充当氧化剂, 在 DMSO 中加热即可完成偶联, 并且此种方法底物适应性 强, 收率较好(Eq. 10).

$$
\begin{gathered}
\mathrm{R}=\frac{\mathrm{CuCl} \text { or } \mathrm{Cu}(\mathrm{OAc})-\mathrm{H}_{2} \mathrm{O}}{\mathrm{DMSO}, \text { air, } 90{ }^{\circ} \mathrm{C}} \mathrm{R} \rightleftharpoons \mathrm{P} \\
\mathrm{R}=\text { Alkyl, Aryl } \quad 65 \% \sim 99 \%
\end{gathered}
$$

2011 年, Stolle 等 ${ }^{[17]}$ 报道了一例有趣的使用振动球 磨机辅助的 Glaser 偶联反应, 此法不需要配体和溶剂, 芳基和烷基取代的端炔在 $\mathrm{KF}-\mathrm{Al}_{2} \mathrm{O}_{3}$ 和催化量的 $\mathrm{CuI}$ 经 过研磨辅助可以高效的实现自偶联给出二炔(Eq. 11), 但通过此法却很难得到单一的非对称二炔.

$$
\mathrm{R} \equiv \frac{\begin{array}{c}
\text { Cul }(5 \mathrm{~mol} \%), \mathrm{KF}_{-} \mathrm{Al}_{2} \mathrm{O}_{3} \\
\text { additional base }
\end{array}}{\begin{array}{c}
\text { zirconia (ball mill) } \\
30 \mathrm{~Hz}, 10 \mathrm{~min} \\
\mathrm{R}=\text { Alkyl, Aryl }
\end{array}} \mathrm{R}=\mathrm{R}
$$

同年, Oishi 等 ${ }^{[18]}$ 报道了利用 $\mathrm{Cu}(\mathrm{OH})_{x}$ 结合氧化锰复 合八面体分子笁体系催化的需氧芳基炔烃的偶联反应 (Eq. 12). 其中氧化锰复合物(OMS-2)不仅起着支持负载 $\mathrm{Cu}(\mathrm{OH})_{x}$ 物种的作用, 而且作为一种电子转移媒介(或称 辅助催化剂)形成一个快速的电子转移通道来加速反应 (Scheme 3).

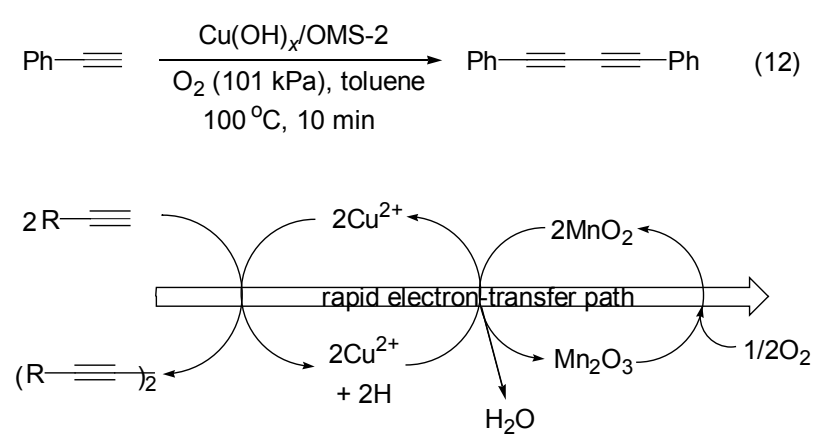

Scheme 3

最近，水相中进行的端炔偶联反应也有报道 ${ }^{[19]}$. 这 类反应使用催化量的 $\mathrm{CuSO}_{4} \cdot 5 \mathrm{H}_{2} \mathrm{O} / \mathrm{TBAB}$ (13)阳离子体 系, $\mathrm{I}_{2}$ 作添加物, 实现了不同类炔烃在水相中的偶联反 应(Eq. 13). 收率良好, 芳香族炔烃较脂肪族炔烃表现出 更优的反应性.

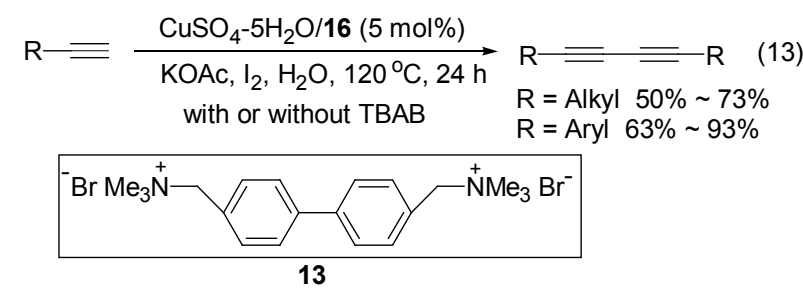

2011 年 Zhang 课题组 ${ }^{[20]}$ 报道了一例改进的 Hay 偶 联方法. 该方法以 $\mathrm{CuI}$ 为铜源、 TMEDA 作配体, 在 3 equiv.三乙胺存在下, 极性溶剂丙酮作溶剂端炔室温下 便可以偶联得到各种不同取代的 1,3-二炔化合物(Eq. 14). 但该反应时间较长，且对两种不同取代的炔只得 到混合的非对称二炔产物.

$$
\begin{aligned}
& \text { Cul (5 mol\%), TMEDA (10 mol\%) } \\
& \mathrm{R}=\frac{\mathrm{Et}_{3} \mathrm{~N} \text { (3.0 equiv.) }}{\text { acetone, air, r.t., } 20 \mathrm{~h}} \mathrm{R}=\mathrm{R} \\
& \begin{array}{l}
R=\text { Alkyl } 73 \% \sim 92 \% \\
R=\text { Aryl } 89 \% \sim 98 \%
\end{array}
\end{aligned}
$$

Jiang 研究组 ${ }^{[21]}$ 将 CuAl-LDH(水滑石)作为催化剂应 用于端炔的均偶联反应中(Eq. 15). 他们还研究了这类 催化剂的循环使用效果以及底物的适应性, 并取得了不 错的结果. 这种 CuAl-LDH 催化剂可以重复利用 9 次而 保持高活性，且反应条件温和，易于操作. 


$$
\begin{aligned}
& \mathrm{R} \equiv \frac{\text { CuAl-LDH, TMEDA }}{\mathrm{CH}_{3} \mathrm{CN} \text {, air, r.t. }} \mathrm{R} \equiv \mathrm{R} \\
& \mathrm{R}=\text { Alkyl, Aryl } \quad 65 \% \sim 88 \%
\end{aligned}
$$

2009 年, Radivoy 等 ${ }^{[22]}$ 在四氢呋喃中仅用 $\mathrm{CuNps}$ (纳 米级颗粒铜粉) 便实现了端炔的偶联反应(Eq. 16). 这种 简洁的体系让人耳目一新, 而且这种催化剂对于低活性 的脂肪族炔烃也有很好的反应性.

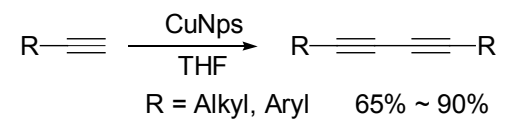

接着在 2011 年, Alonso 等 ${ }^{[23]}$ 报道了 $\mathrm{CuNps} / \mathrm{TiO}_{2}$ 催 化的端炔偶联反应(Eq. 17), 该反应仅需 $1 \mathrm{~mol} \%$ 催化剂 用量, 以哌啶作碱在四氢呋喃作溶剂或无溶剂条件下均 可实现不同取代炔基均偶联反应. 另外该催化剂也可以 循环利用, 无论是空气或惰性气体氛围中都表现出良好 的催化性能.

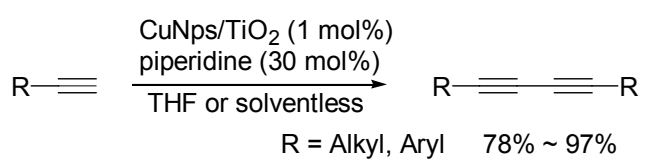

最近, 一种固体附载的 $\mathrm{Cu}(\mathrm{OH})_{x} / \mathrm{TiO}_{2}$ 非均相催化剂 被证明能有效地催化端炔氧化偶联反应(Eq. 18) ${ }^{[24]}$. 芳 香族和脂肪族端炔均可以以较好的收率得到二炔化物. 而且研究表明, 仅将 $\mathrm{Cu}(\mathrm{OH})_{2}$ 和 $\mathrm{TiO}_{2}$ 简单混合并不能起 到催化效果.

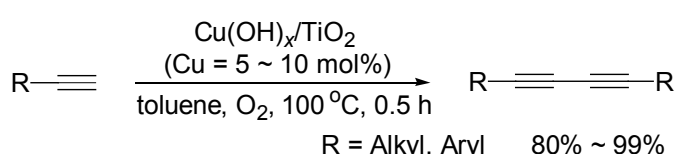

2011 年, Balamurugan 研究组 ${ }^{[25]}$ 报道了一例 $\mathrm{CuCl}_{2}$ 催化、DBU 作碱的端炔对称和非对称偶联反应(Scheme 4). 通过使其中一种贫电子炔过量可以得到不对称的二 炔化物，但此法对脂肪族端炔效果并不理想.

$$
\begin{aligned}
& \mathrm{R} \equiv \frac{\mathrm{CuCl}_{2} \text { (10 mol\%), DBU (1.2 equiv.) }}{\mathrm{THF}, \text { r.t., } 24 \mathrm{~h}} \mathrm{R}=\mathrm{Aryl} \underset{\overline{65 \%} \sim 99 \%}{\bar{\equiv}} \mathrm{R} \\
& \mathrm{Ph}=+\equiv \mathrm{OH} \frac{\mathrm{CuCl}_{2} \text { (10 mol\%), DBU (1.2 equiv.) }}{\text { THF, r.t., } 24 \mathrm{~h}} \\
& \mathrm{Ph}=\mathrm{OH}
\end{aligned}
$$

\section{Scheme 4}

2010 年, Kesavan 等 ${ }^{[26]}$ 发展了一类室温条件下即可 进行的端炔偶联反应，它使用 $\mathrm{Cu}(\mathrm{OAc})_{2}-\mathrm{H}_{2} \mathrm{O}$ 作催化剂, 加入化学计量的哌啶, 以二氯甲烷为溶剂, 几乎定量地
得到对称的 1,3-二炔. 而且, 通过改变底物物质的量比 可以得到非对称的二炔化物(Eq. 19).

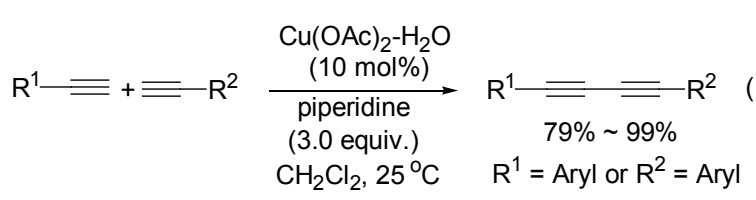

前不久, Shibata 小组 ${ }^{[27]}$ 报道了一例新奇的 Hay 偶联 反应。他们使用一种清洁剂 solkane ${ }^{\circledR} 365 \mathrm{mfc}\left(\mathrm{CF}_{3} \mathrm{CH}_{2-}\right.$ $\mathrm{CF}_{2} \mathrm{CH}_{3}$ ) 代替常用的有机溶剂实现了此类 Hay 偶联反应 (Eq. 20). 该反应在室温下即可进行，而且反应时间非常 短，底物适应性好，不失为一种端炔偶联的好方法.

$$
\begin{aligned}
& \mathrm{R}=\frac{\mathrm{CuCl}(10 \mathrm{~mol} \%), \text { TMEDA }(10 \mathrm{~mol} \%)}{\text { Sokane }^{\circledR} 365 \mathrm{mfc} \text {, air, r.t. }} \mathrm{R} \equiv \mathrm{R}(20) \\
& \mathrm{R}=\text { Alkyl, Aryl } \quad 82 \% \sim 98 \%
\end{aligned}
$$

纵观这几年的铜试剂催化的端炔偶联反应不难发 现，大部分还是在早期研究基础上的改进和修饰，并且 逐渐朝着简单化、操作方便和条件温和的方向发展.

\section{2 钯催化铜促进的端炔偶联反应}

自 1985 年 Rossi 等 ${ }^{[28]}$ 把 Pd 试剂引入到端炔的均偶 联反应中以来，以 $\mathrm{Pd}$ 催化 $\mathrm{Cu}$ 促进的端炔偶联反应以其 广泛的底物耐受性和相对较低的催化剂用量引起了人 们的广泛关注.

1997 年, Liu 和 Burton ${ }^{[29 a]}$ 发展了一类重要的 Pd 催 化 $\mathrm{Cu}$ 促进的端炔偶联反应. 他们使用 $\left[\mathrm{PdCl}_{2}\left(\mathrm{PPh}_{3}\right)_{2}\right]$ 和 $\mathrm{CuI}$ 作催化剂, $\mathrm{I}_{2}$ 作氧化剂, 在 $i-\mathrm{Pr}_{2} \mathrm{NH}$ 存在下以较好的 收率拿到了 1,3-共轭二炔产物(Eq. 21). 而且无论是芳香 族炔烃和脂肪族炔烃都取得了良好的结果. 2009 年 Kijima 等 ${ }^{[29 b]}$ 把这类方法应用到炔聚合物的合成中.

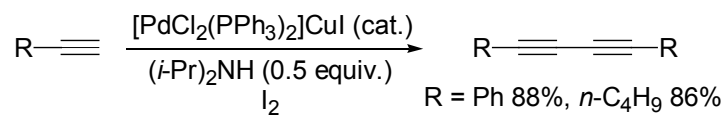

Najera 等 ${ }^{[30]}$ 将首次将环状肜类 Pd 络合物 14 和 15 应用到卤代芳烃端炔的偶联反应中．随后，他又将此类 环状肜类 Pd 络合物应用到 Glaser 类偶联反应中, 取得 了不错的结果(Eq. 22) ${ }^{[31]}$. 该反应在不影响产率的前提 下，催化剂的用量可降至 $0.05 \mathrm{~mol} \%$.

$$
\begin{aligned}
& \mathrm{R}=\frac{\text { Pd-cat. } 14 \text { or } 15(0.05 \sim 0.5 \mathrm{~mol} \%)}{\text { Pyrrolidine or TBAA }(1.1 \text { equiv. })} \mathrm{R}=\mathrm{R} \\
& \text { Cul (5 mol\%), NMP } \mathrm{R}=\text { Alkyl, Aryl } 63 \% \sim 99 \%
\end{aligned}
$$

2007 年, Shreere 等 ${ }^{[11]}$ 将第 1.1 节中所描述的类似的 碱性离子液体 16 应用到 $\mathrm{Pd}$ 催化的端炔偶联反应中同样 


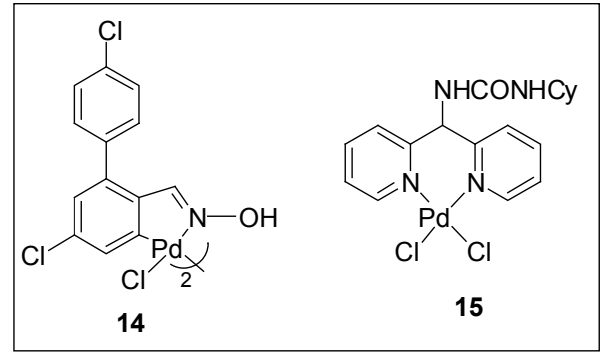

适用(Eq. 23). 与常用有机溶剂相比, 该类离子液体对此 类炔偶联体系有更好的溶解性, 因此能够更好的促进反 应.

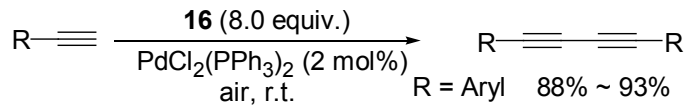

$$
\begin{aligned}
& \mathrm{X}^{-} \quad \mathrm{X}=\mathrm{NTf}_{2}
\end{aligned}
$$

同年 Jiang 等 ${ }^{[32]}$ 报道了利用容易制备的聚合物固载 $\mathrm{Pd}$ 配合物和 $3 \mathrm{~mol} \% \mathrm{CuI}$ 在水相中完成的 Glaser 偶联反 应. 碳酸钠作碱, $\mathrm{Pd}$ 试剂充当氧化剂, 通过此法可以以 中等收率得到二炔(Eq. 24).

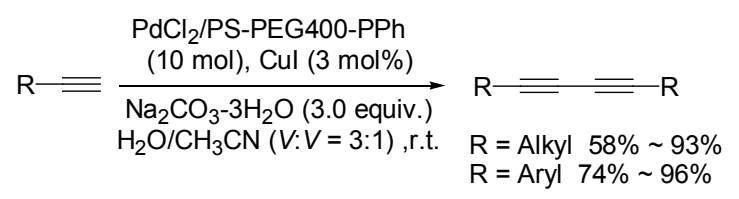

随后 Sajiki 等 ${ }^{[33]}$ 报道了一例 $\mathrm{Pd} / \mathrm{C}$ 和 $\mathrm{CuI}$ 催化的非 均相偶联反应. 简洁的催化系统、温和的反应条件和普 遍的底物适用性是该反应的优点(Eq. 25).

$$
\mathrm{R} \equiv \frac{\begin{array}{c}
\mathrm{Pd} / \mathrm{C}(0.03 \sim 1 \mathrm{~mol} \%) \\
\text { Cul }(3 \mathrm{~mol} \%)
\end{array}}{\mathrm{DMSO}, \text { air, r.t. }} \longrightarrow \mathrm{R}=\begin{aligned}
& \mathrm{R}=\text { Alkyl } 81 \% \sim 99 \% \\
& \mathrm{R}=\text { Aryl } 91 \% \sim 98 \%
\end{aligned}
$$

2009 年, Watarai 研究组 ${ }^{[34]}$ 合成了一类 N-杂卡宾大 环螯合物催化剂, 并把它们应用到芳基炔烃的偶联反应 中. 实验显示这类催化剂具有一定的催化效果(Eq. 26).

最近 Perrone 等 ${ }^{[35]}$ 报道了 $\mathrm{Pd}(\mathrm{OAc})_{2}$ 催化的几乎可以 定量转化的苯乙炔自偶联反应, 遗憾的是此方法并没能 扩展到其他类型的端炔底物中(Eq. 27).

2011 年, $\mathrm{Li}$ 等 ${ }^{[36]}$ 发展了一类 $\mathrm{PdCl}_{2}\left(\mathrm{PPh}_{3}\right)_{2} / \mathrm{CuI}$ 催化 的端炔偶联反应. 19 作为新型的氧化剂来实现这一类 Glaser 偶联反应，以中等收率得到二炔产物(Eq. 28). 同 样，这种方法也可以应用到炔基硅烷的自偶联反应中. 以无机碱碳酸钾代替 Pd 催化中常用的有机碱是该反应 的特点.
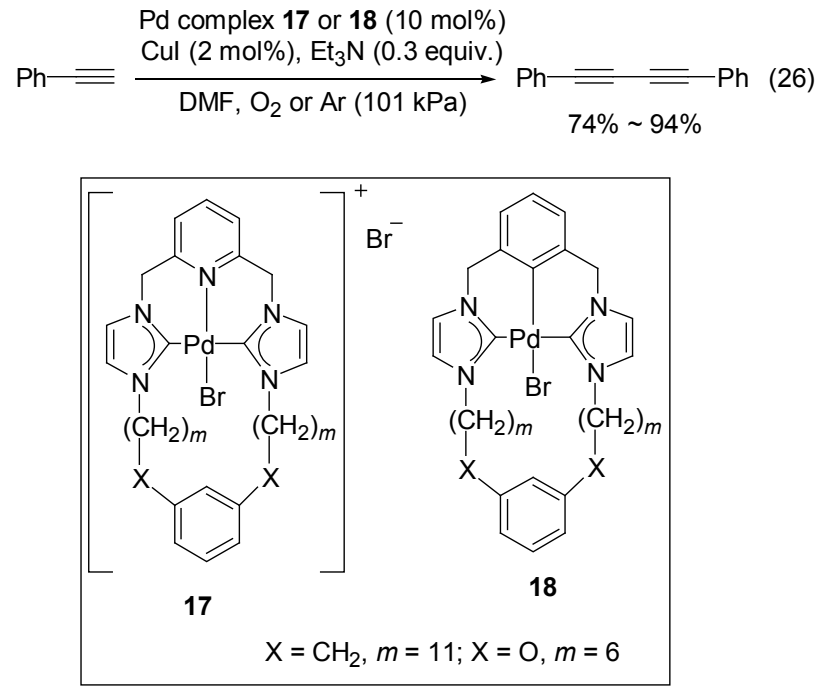

$$
\begin{aligned}
& \mathrm{Ph}=\frac{\mathrm{Pd}(\mathrm{OAc})_{2}, \mathrm{Ph}_{3} \mathrm{P}, \mathrm{CH}_{3} \mathrm{CH}_{2} \mathrm{Cl}}{\mathrm{CO}(2.76 \mathrm{MPa}), \mathrm{Et}_{3} \mathrm{~N}} \mathrm{Ph} \equiv \bar{\equiv} \\
& \mathrm{THF}, 110^{\circ} \mathrm{C}, 14 \mathrm{~h}
\end{aligned}
$$

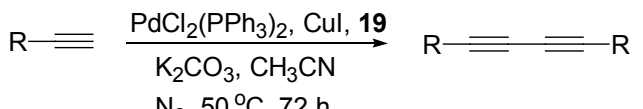
$\mathrm{N}_{2}, 50^{\circ} \mathrm{C}, 72 \mathrm{~h}$

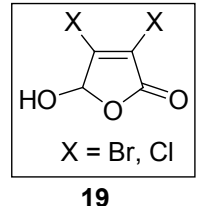

\section{3 其他金属参与的端炔偶联反应}

近年来, 除了主要的 $\mathrm{Cu}$ 试剂和 $\mathrm{Pd}$ 试剂催化端炔偶 联反应之外，其他金属参与的端炔偶联反应也有报道. Bharathi 等 ${ }^{[37}$ 报道了 $\mathrm{TiCl}_{4}$ 催化的端炔偶联反应，虽然该 反应操作方便, 但产率却不尽人意. 作者在文章中提出 炔烃与金属形成过渡态 20, 20 经过还原消除得到 1,3-二 炔(Scheme 5).

$$
\begin{aligned}
& \mathrm{R}=\frac{\mathrm{Ti}_{4} \mathrm{Cl} / \mathrm{Et}_{3} \mathrm{~N}}{\mathrm{CH}_{2} \mathrm{Cl}_{2}, 0 \sim 25^{\circ} \mathrm{C}, 6 \mathrm{~h}} \underset{\mathrm{R}}{\stackrel{\mathrm{Cl}}{\longrightarrow}} \\
& \mathrm{R}=\mathrm{R} \quad \mathrm{R}=\text { Alkyl, Aryl } \quad 43 \% \sim 67 \%
\end{aligned}
$$

\section{Scheme 5}

之后 Krafft 课题组 ${ }^{[38]}$ 研究发现, 一氧化碳和钴的络 合物可以催化端炔偶联得到 1,3-二炔(Eq. 29) ${ }^{[38]}$. 作者 在文中指出在碱存在下, $\mathrm{Co}_{2}(\mathrm{CO})_{8}$ 经过配体交换和歧化 反应生成 HNIP(同核离子对), 实际起催化作用的是该 离子对. 


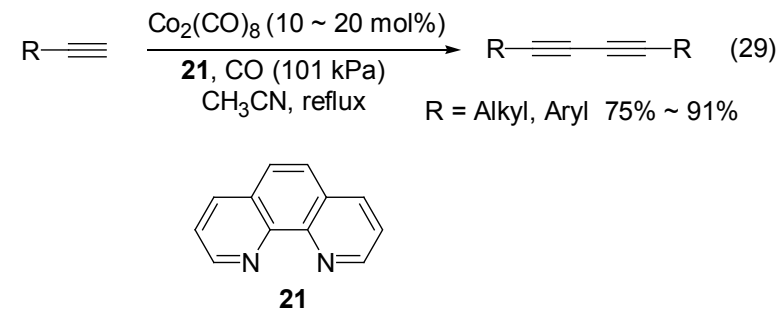

2008 年, 另一种钴试剂催化的端炔偶联反应被发 现 ${ }^{[39]}$. 以化学计量的硝基苯为氧化剂在 $10 \mathrm{~mol} \% \mathrm{CoBr}_{2}$, $\mathrm{ZnI}_{2}$ 和 1 equiv. 锌粉存在下可以以中等至良好的收率得 到二炔化合物(Eq. 30), 文中还给出了可能的反应机理 (Scheme 6).

$$
\mathrm{R}=\frac{\mathrm{CoBr}_{2}(10 \mathrm{~mol} \%), \mathrm{Znl}_{2}(10 \mathrm{~mol} \%)}{\mathrm{Zn} \mathrm{powder}, \mathrm{PhNO}_{2}} \mathrm{R}=\mathrm{R} \text { (30) }
$$$$
\mathrm{R}=\mathrm{Ph} 99 \%, \mathrm{Me}_{3} \mathrm{Si} 56 \%, \mathrm{C}_{4} \mathrm{H}_{9} 52 \%
$$
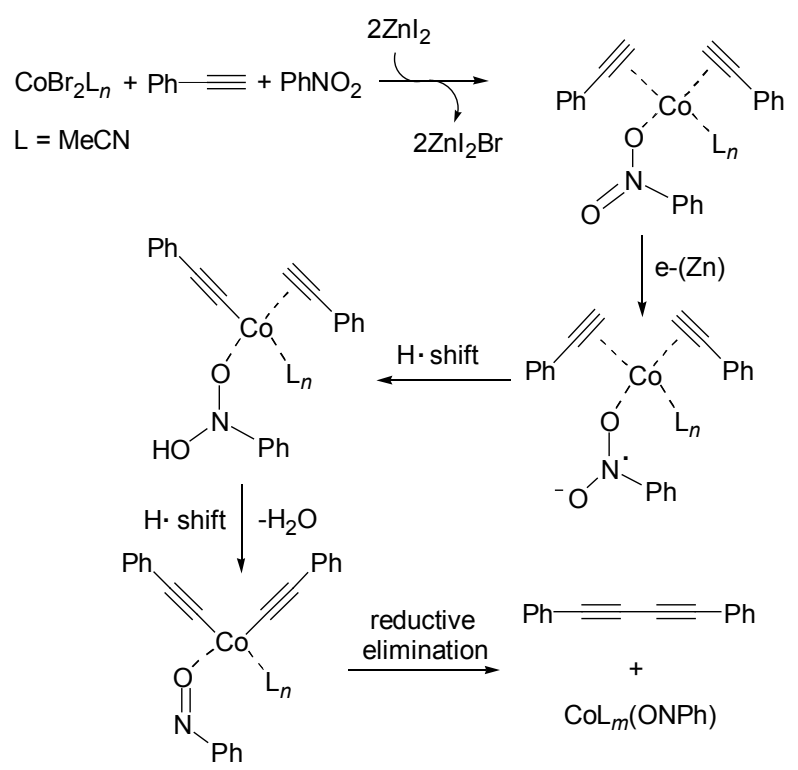

Scheme 6

2009 年 Lei 研究组 ${ }^{[40]}$ 报道了第一例 $\mathrm{Ni}$ 盐催化的端 炔偶联反应(Eq. 31). 该反应不仅应用于各类端炔的均 偶联反应, 而且通过改变两种炔烃的物质的量比可以实 现不同炔烃之间的非对称偶联.

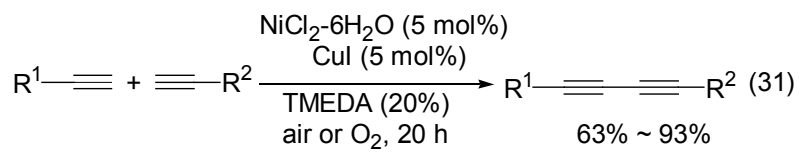

最近 Kishi 小组 ${ }^{[41]}$ 又将 $\mathrm{Ni}$ 配合物应用到碘代炔的偶 联反应中(第 2.1 节). 除此之外, 关于 $\mathrm{Fe}$ 试剂和 $\mathrm{Mn}$ 试剂 催化的炔基格氏试剂的偶联反应将在第 2.1 节中予以介 绍.

Mizuno 等[42a] 将 ${ }^{2 B A_{4}}\left[\mathrm{r}-\mathrm{H}_{2} \mathrm{SiW}_{10} \mathrm{O}_{36} \mathrm{Cu}_{2}\left(\mu-1,1-\mathrm{N}_{3}\right)_{2}\right]$
作为催化剂运用到不同结构类型的端炔偶联反应中. 氧 气条件下, 以苯甲腈为溶剂, 只需 $2.2 \mathrm{~mol} \%$ 催化剂即可 完成反应. 2011 年, 该小组 ${ }^{[42 b]}$ 又报道了一例类似的反 应. 这类催化剂可以回收利用并有很好的官能团适应性 (Eq. 32).

$$
\begin{array}{r}
\mathrm{R} \rightleftharpoons \frac{\text { Silicotungstate complex }}{\text { Benzonitrile, } \mathrm{O}_{2}, 100^{\circ} \mathrm{C}} \mathrm{R}=\frac{\overline{=}}{\mathrm{R}}=\mathrm{Alkyl}, \text { Aryl } 76 \% \sim 97 \%
\end{array}
$$

\section{2 炔类衍生物的偶联反应}

Glaser 类型的端炔氧化偶联反应多给出对称的 1,3二炔产物，对于非对称的二炔化物结果却不如人意. 而 炔类衍生物的交叉偶联反应是合成非对称二炔的主要 方法.

\section{1 炔基卤代物的偶联反应}

早在 1957 年 Cadiot 和 Chodkiewicz 等[43a,43b]便发展 了经典的 Cadiot-Chodkiewicz 交叉偶联反应. 该反应是 指在一价铜盐和脂肪族胺存在下，1-澳代炔和端炔发生 的杂偶联反应(Eq. 33). 自此便拉开了合成非对称共轭 二炔化合物的序幕. 近年来，这些方面的报道也有很多.

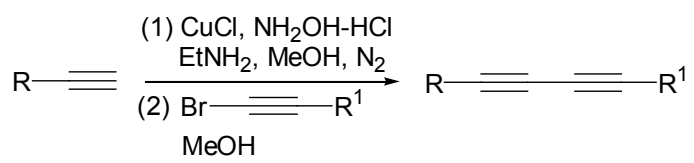

Shen 等 ${ }^{[4]}$ 报道了一例 Pd 催化的二溴代烯来制备对 称二炔和非对称二炔的方法(Scheme 7). 对于形成对称 二炔需要一个弱的配体 TFP, 另外催化量的 CuI 可以加 速反应进行; 而要生成不对称的二炔则需要提供一个富 电子的配体(4-MeOPh) $)_{3} \mathrm{P}$. 该反应过程中会生成少量的 副产物 22 或 23. 作者还给出了可能的反应机理(Scheme 8).

2008 年, Lei 小组 ${ }^{[5]}$ 尝试使用缺电子烯烃膦配体 L 为配体促进 Pd 催化的溴代炔和端炔的交叉偶联反应, 取得了很好的效果(Eq. 34). 此方法不失为一种制备非 对称二炔的好方法. 它填补了 Glaser 和 ChodkiewiczCadiot 类偶联反应的不足，作者在文中还给出了该催化 反应机理(Scheme 9).

$$
\mathrm{R}^{1}=\mathrm{Br}+\equiv \mathrm{R}^{2} \frac{\substack{\mathrm{Pd}(\mathrm{dba})_{2} / \mathrm{L} \\ \mathrm{EM}{ }_{3} \mathrm{~N}, \mathrm{Cul} \\ 2 \sim 9 \mathrm{~h}, \mathrm{r.t.}}}{\stackrel{\mathrm{L}}{\longrightarrow}} \mathrm{R}^{1}=\mathrm{R}^{2}
$$




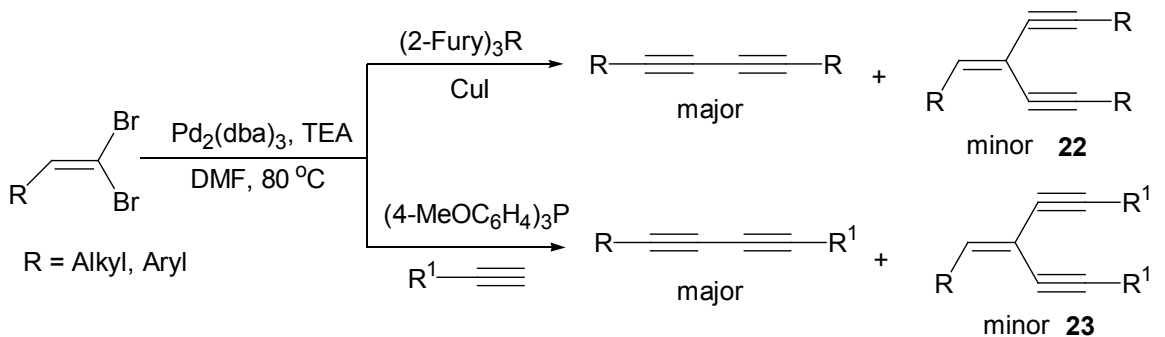

Scheme 7
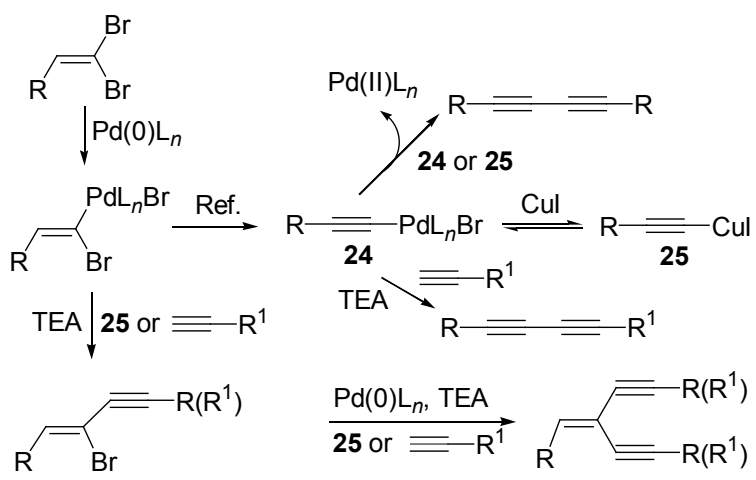

Scheme 8

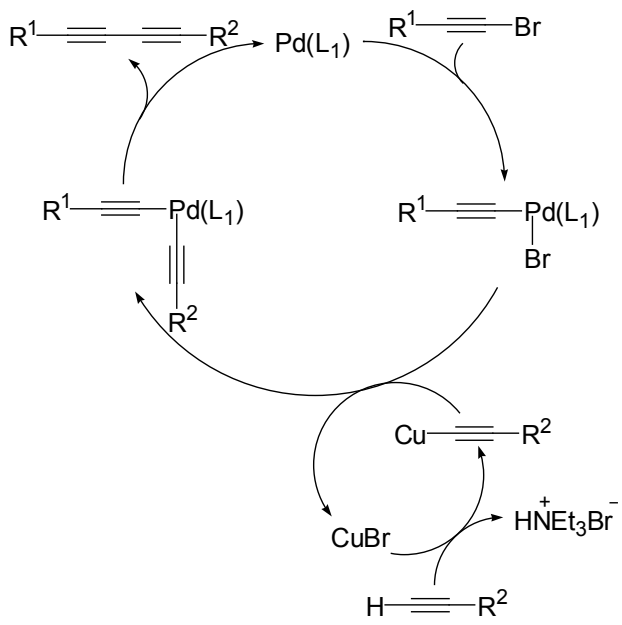

Scheme 9

碘代炔的偶联反应同样可以通过 Pd 催化实现, Lee 等 ${ }^{[46]}$ 发现以 $\mathrm{Pd}\left(\mathrm{PPh}_{3}\right)_{4}$ 作催化剂在 DMF 中可以实现碘代 炔的自偶联反应(Eq. 35). 该反应在室温下即可以以较 好收率得到偶联产物. 底物中的取代基电子效应对此反 应的影响并不明显. 而且对于通常情况下反应性能不佳 的脂肪族炔烃也表现出较好的反应性. 该反应以零价钯 的催化循环机理进行(Scheme 10).

$$
\mathrm{R}=\mathrm{I} \underset{\mathrm{N} 2 \text {, DMF, r.t. }}{\stackrel{\mathrm{Pd}\left(\mathrm{PPh}_{3}\right)_{4}(4 \mathrm{~mol} \%)}{\longrightarrow}} \mathrm{R}=\frac{\mathrm{Alkyl} \text {, Aryl } \quad 75 \% \sim 95 \%}{=}
$$

之后, Xue 等 ${ }^{[47]}$ 发展了一类铜粉催化的芳基碘代炔

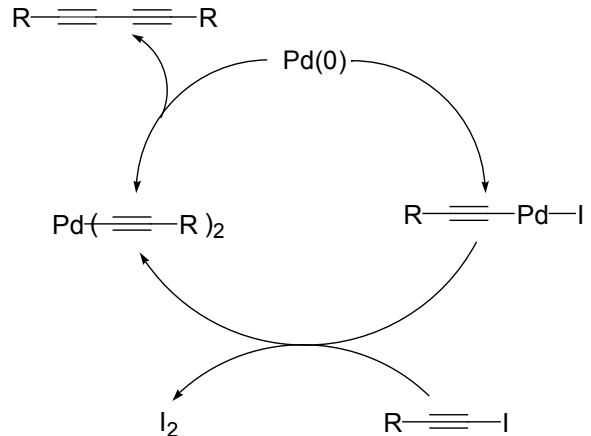

Scheme 10

的自偶联反应(Eq. 36). 反应使用等量的铜粉和吡啶，但 反应使用了有毒的苯做溶剂，而且遗憾的是对于脂肪族 的磑代炔效果并不理想.

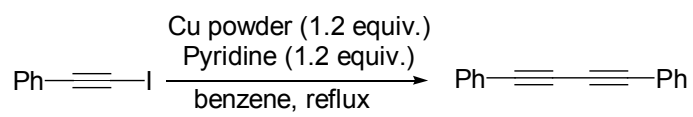

2011 年, Kishi 研究组 ${ }^{[41]}$ 运用 Ni 配合物 25 和 $\mathrm{Zr}(\mathrm{Cp})_{2} \mathrm{Cl}_{2}$ 实现了碘代炔的自偶联反应(Eq. 37), 此外需 要加入 2 equiv. $\mathrm{Mn}$ 和 $\mathrm{LiCl}$. 作为一种新方法, 该方法有 广泛的底物适应性.

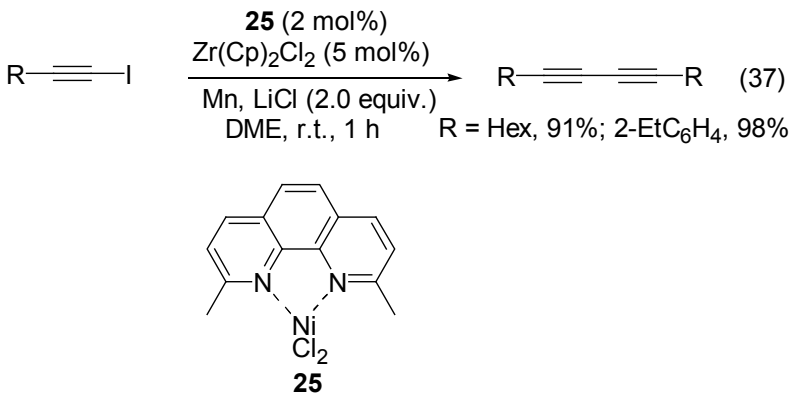

最近, Chen 等 ${ }^{[48]}$ 发展了一种简单的无需金属催化 的炔基卤代物的偶联反应(Eq. 38). 该反应中，炔基卤代 物与 3 equiv. KI 试剂在 DMF 中加热至 $120{ }^{\circ} \mathrm{C}$ 反应若干 小时就可生成相应的 1,3-二炔化合物. 整个过程操作方 便、无需碱和金属，作者给出了可能的反应机理(Scheme 11). 


$$
\underset{\mathrm{X}=\mathrm{Br}, \mathrm{I}}{\stackrel{\mathrm{DMF}}{\rightleftharpoons} \mathrm{XI}} \underset{\mathrm{R}=\text { Alkyl, Aryl } \quad 50 \% \sim 99 \%}{\mathrm{R}} \mathrm{R}
$$

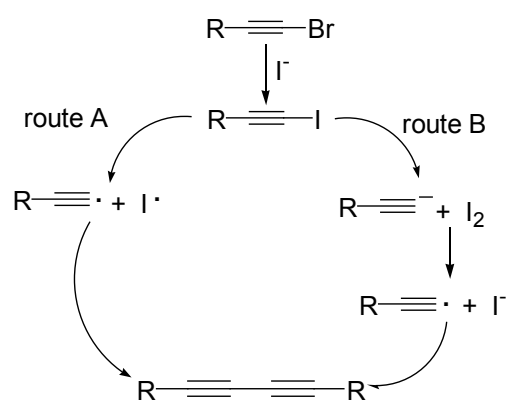

Scheme 11

2009 年 Cahiez 等 ${ }^{[49]}$ 研究了一类 Mn 试剂催化的炔 基氯化镁之间的交叉偶联反应(Eq. 39). 这也是第一例 金属催化的两种格氏试剂之间的偶联反应, 该反应中均 偶联和交叉偶联通常取决于底物格氏试剂的性质.

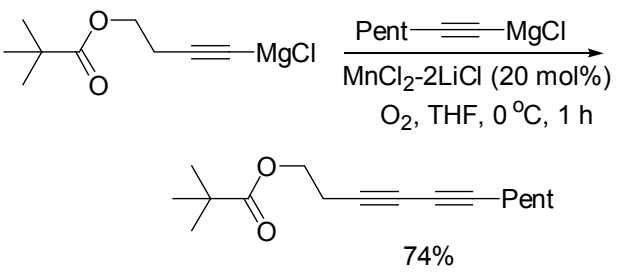

最近, Itoh 等 ${ }^{[50]}$ 报道了一类 $\mathrm{FeCl}_{3}$ 催化的芳基格氏试 剂之间的偶联反应(Eq. 40). 值得一提的是, 该反应使用 了 $\left[\mathrm{P}_{444} \mathrm{ME}\right]\left[\mathrm{NTf}_{2}\right]$ 溶剂系统, 且该反应速度非常快, 在 $30 \mathrm{~min}$ 内即可完成. 温和的反应条件和较低的催化剂用 量是该反应的优点.

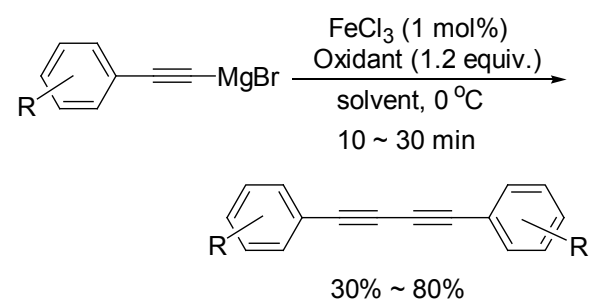

\section{2 炔基硅烷的偶联反应}

炔基硅烷也可以作为底物参与铜盐催化的炔烃偶 联反应. Hiyama 研究组 ${ }^{[51]}$ 发现在温和条件下, 以极性溶 剂 $\mathrm{DMF}$ 为媒介, 炔基硅烷可以通过等量 $\mathrm{CuCl}$ 作用得到 自偶联的二炔产物(Eq. 41). 同时他还发现炔基硅烷与 氯代炔在催化量 $\mathrm{CuCl}$ 作用下形成非对称二炔(Eq. 42).

$$
\begin{array}{r}
\mathrm{R}=-\mathrm{SiMe}_{3} \frac{\mathrm{CuCl}}{\mathrm{DMF}, 60^{\circ} \mathrm{C}} \mathrm{R}=\mathrm{R} \\
\mathrm{R}=\mathrm{Ph}, 99 \% ; n-\mathrm{C}_{6} \mathrm{H}_{13}, 80 \%
\end{array}
$$

$$
\begin{gathered}
\mathrm{R}^{1}=\mathrm{SiMe}_{3}+\mathrm{R}^{2}=\mathrm{Cl} \quad \frac{\mathrm{CuCl}(10 \mathrm{~mol} \%)}{\mathrm{DMF}, 80^{\circ} \mathrm{C}} \\
\mathrm{R}^{1}=\overline{\overline{ }}=\mathrm{R}^{2} \\
\mathrm{R}^{1}, \mathrm{R}^{2}=\text { Aryl } \quad 62 \% \sim 97 \%
\end{gathered}
$$

之后 Kunai 等 ${ }^{[52]}$ 在催化量 $\operatorname{Pd}(\mathrm{OAc})_{2}$ 和 $\mathrm{dppp}$ 为配体 的条件下完成了一例炔基硅化合物的自偶联反应(Eq. 43), 不过，该反应产率偏低，即使是对于活性较高的苯 基取代的炔硅化物，产率也只有 $70 \%$.

$$
\begin{gathered}
\mathrm{R}=\mathrm{SiMe}(\mathrm{OMe})_{2} \frac{\mathrm{Pd}(\mathrm{OAc})_{2}(5 \mathrm{~mol} \%), \mathrm{dppp}(7.5 \mathrm{~mol} \%)}{\mathrm{DMSO}, 50^{\circ} \mathrm{C}} \\
\mathrm{R}=\mathrm{R}
\end{gathered}
$$

$\mathrm{R}=\mathrm{Ph}, 70 \%$; 1-Naphthyl, 57\%; 1-Cyclohexenyl, 50\%

2011 年, Stolle 小组 ${ }^{[17]}$ 报道了一例苯基炔硅化物偶 联反应. 该反应以 TMS 保护苯乙炔并通过氟化物原位 脱保护转化为偶联产物(Eq. 44).

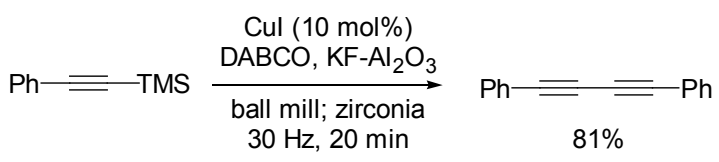

\section{3 炔基硼烷的偶联反应}

Reddy 等 ${ }^{[53]}$ 报道了炔基硼的锂盐参与的炔偶联反 应. 底物在 $\mathrm{Pd}(\mathrm{OAc})_{2} / \mathrm{DPEPhos}$ 催化下, 无需碱和氧化 剂, 以 $\mathrm{CuI}$ 作为共催化剂的条件下以良好的收率得到二 炔产物(Eq. 45). 底物中包含贫电子和富电子基团以及 各种保护基团都能得到不错的结果.

$$
\begin{aligned}
& {\left[\mathrm{R}=\mathrm{B}(\mathrm{OPr}-\mathrm{i})_{3}\right]^{-} \mathrm{Li}^{+} \stackrel{\begin{array}{c}
\mathrm{Pd}(\mathrm{OAC})_{2}(0.05 \mathrm{~mol} \%) \\
\mathrm{DPEPhos}(0.05 \mathrm{~mol} \%)
\end{array}}{\mathrm{Cul}, \mathrm{THF}, 60^{\circ} \mathrm{C}}} \\
& \mathrm{R}=\mathrm{Alkyl}, \text { Aryl } \quad 63 \% \sim 93 \%
\end{aligned}
$$

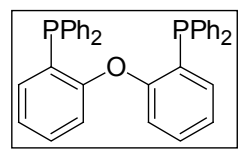

随后 Nishihara 等 ${ }^{[54]}$ 报道了另一类炔基硼化物参与 的偶联反应(Eq. 46). 在极性溶剂 DMF 中, 用等量的 $\mathrm{Cu}(\mathrm{OAc})_{2}$ 来促进该反应，该法由于其官能团耐受性好 而成为一种重要的形成 $\mathrm{C}_{\mathrm{sp}}-\mathrm{C}_{\mathrm{sp}}$ 键的方法.

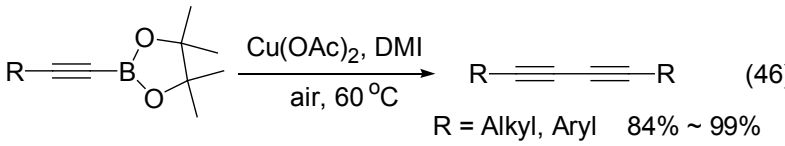

\section{4 其它炔类化合物的偶联反应}

除了以上常见的几种炔衍生物的偶联反应外，炔基 锡试剂也可以进行此类反应. Hiyama 等 ${ }^{[55]}$ 报道了 $\mathrm{Pd}$ 试 剂催化的炔基锡化物参与的均偶联反应(Eq. 47). 该反 
应以 26 为配体, $\mathrm{CH}_{2}=\mathrm{CHCH}_{2} \mathrm{OAc}$ 作为氧化剂在反应过 程中被还原。

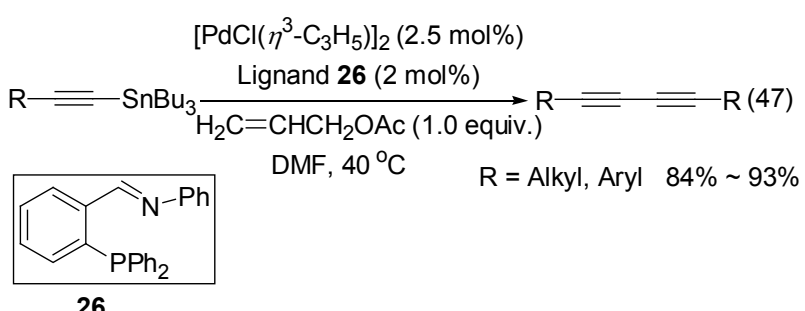

随后, Ochiai 小组 ${ }^{[56]}$ 发展了一类通过 $\mathrm{BF}_{3} \cdot \mathrm{Et}_{2} \mathrm{O}$ 处理 二氟溴苯和炔基锡化物制备二炔的方法，作者发现当存 在过量炔基锡化物( $>2.0$ equiv.) 时可以得到炔偶联产物 (Scheme 12). 有趣的是, 这类反应只适用于通常反应性 不佳的脂肪族炔烃.

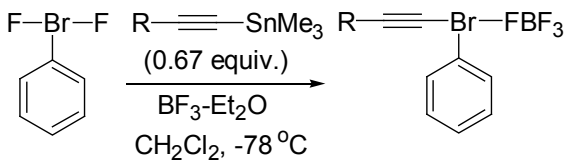

$\mathrm{R}=\mathrm{D}$

$\mathrm{R}=$ Alkyl, $72 \%$ 87\%; Ph, 33\%

\section{Scheme 12}

最近, Stefani 等 ${ }^{[57]}$ 发现在超声波辅助下, 以炔基碲 化物为原料, 经过 $\mathrm{Pd}$ 催化可以得到偶联产物(Eq. 48), 而且此方法有很好的官能团适应性, 是端炔自偶联反应 的一个很好的补充.

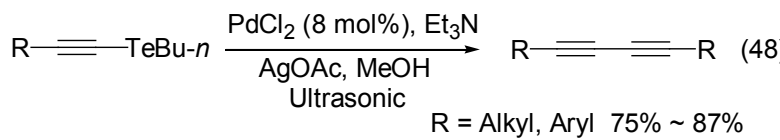

\section{3 机理研究}

虽然近些年多种多样的催化体系陆续被报道, 但在 机理研究方面却鲜有人问津, 许多文章仅提供了一些假 设的反应机理. 上面两部分中也有涉及到部分机理. 目 前为止, 这方面较多的还是关于传统的氧化偶联反应机 理描述, 它们准确详尽的机理目前尚不清楚, 现就几类 普遍接受的机理举例说明.

\section{1 铜试剂催化的炔偶联机理}

早在 1936 年, Zalkind 和 Fundyler ${ }^{[58 a, 58 b]}$ 就提出了铜 盐单电子转移的自由基机理. 之后 1963 年 Clifford 和 Water ${ }^{[59]}$ 发展了此类自由基偶联机理，它们认为：端炔 在碱作用下先形成炔铜中间体, 然后在醋酸配体促进下 很快通过单电子转移给二价铜而被氧化给出自由基单 体, 最后发生自由基自聚形成二炔产物. 这类自由基机
理在当时被人们所认可(Scheme 13) ${ }^{[4]}$.

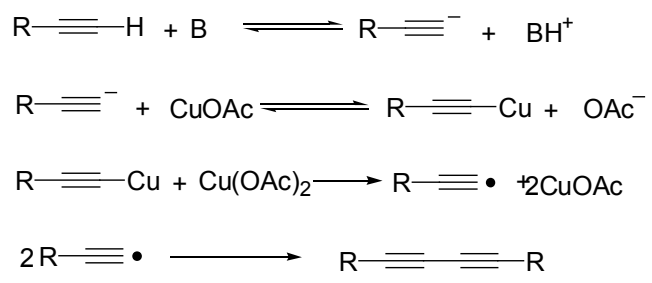

Scheme 13

之后 1964 年, Bohlmann 等 ${ }^{[60]}$ 做了一系列电子效应 对反应速率影响的实验发现, 酸性越强的炔在碱性条件 下自偶联越快; 然而在酸性条件下结果却恰好相反. 而 且添加的一价铜盐对反应至关重要. 因此他对早期提出 的自由基机理提出了质疑，并提出了通过形成金属一炔 键的 $\pi$-配合物来活化炔烃的猜想，同时还首次提出了双 核炔铜配合物中间体的机理(Scheme 14) ${ }^{[7]}$. 碱作用下, 一价铜盐与端炔形成炔铜配合物 27 和多配位铜配合物 28, 之后转化为双核炔铜配合物 29 , 最后分解给出偶联 的二炔产物. 这类机理代替早期的自由基机理被人们广 泛认可和接受. 但此机理并不能圆满解释以氧气为氧化 剂的 Glaser 偶联反应.

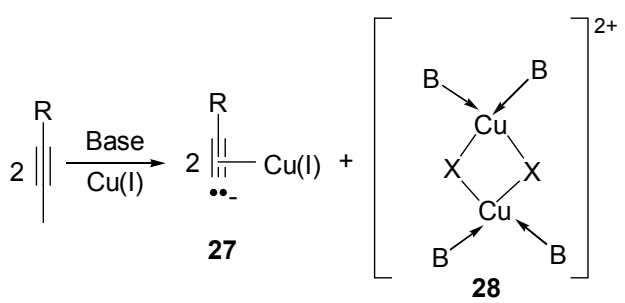

28

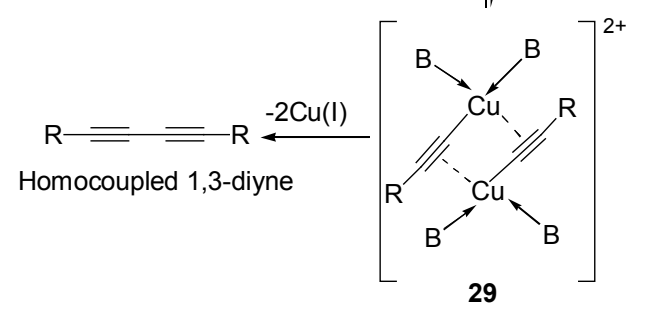

Scheme 14

之后许多研究者还提出了一价铜配合物在内的其 它一些可能的机理, 也能解释一些实验结果,但都未被 人们广泛接受 ${ }^{[4 a]}$.

Cadiot 等 ${ }^{[43 a]}$ 发展的另外一类经典的交叉偶联反应 并提出了相应的反应机理. 该机理认为反应经过一价炔 铜化物 30 与卤代炔氧化加成形成中间体三价铜物种 $\mathbf{3 1}$, 而后还原消除得到非对称二炔(Scheme 15) ${ }^{[4 a]}$.

总之, 铜试剂催化的端炔偶联反应机理很大程度上 取决于反应条件, 然而目前被普遍认可的仍是第 3.1 节 中所述 Bohlmann 等提出的机理 ${ }^{[60]}$. 


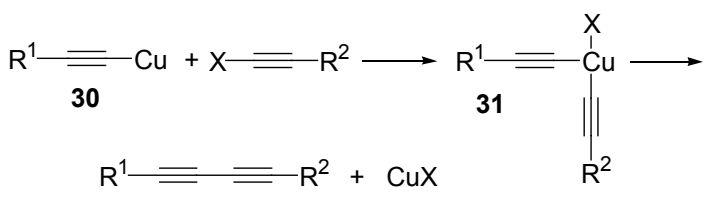

Scheme 15

\section{2 钯试剂催化的炔偶联反应}

最近研究比较多的 $\mathrm{Pd}$ 催化 $\mathrm{Cu}$ 促进的炔烃偶联反应 多遵循 1989 年 Alper ${ }^{[61]}$ 提出的 $\operatorname{Pd}(0)-P d(I I)$ 循环机理. 本 文以 1997 年 Liu 等 ${ }^{[2]}$ 给出的此类循环机理为代表简单 介绍(Scheme 16). 首先, $I_{2}$ 作用下, 二配位的零价钯活 性中间体转化为二价钯, 炔化物经 $\mathrm{CuX}$ 活化后与二价 钯形成二炔钯中间体 32, 最后还原消除得到二炔, 同时 释放出活性零价钯再次进入循环催化反应.

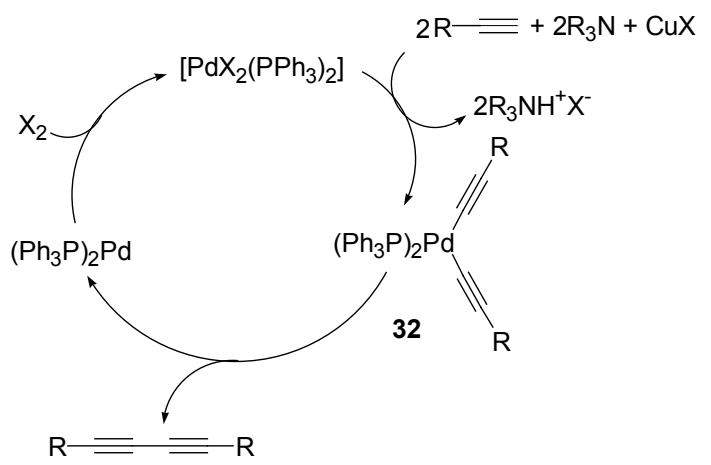

Scheme 16
至于 Pd 催化的交叉偶联反应机理的研究始于 1984 年 Bugamin ${ }^{[63]}$ 等. 本文以 1995 年 Cai 和 Vasella ${ }^{[64]}$ 提出 的催化循环为代表做简单介绍(Scheme 17) ${ }^{[4 a]}$. 炔卤化 物与零价钯氧化加成得到 $\eta^{2}-\mathrm{Pd}$ 配合物 $\mathbf{3 3}, \mathbf{3 3}$ 在一价铜 作用下重排为 34, 34 快速转化为双金属配合 35. 炔铜化 物转金属化形成二炔化物 36, 最后给出终产物. 这种机 理也被人们普遍认可.

最近, Lei 研究组 ${ }^{[45]}$ 在文章中归纳了 Pd 催化的交叉 偶联反应的三种途径(Scheme 18). 他还指出通过控制 还原消除步骤可以选择性的得到不同类型的非对称二 炔化物.

\section{4 炔偶联反应的应用}

炔偶联反应广泛应用于各类炔化物的合成、天然产 物合成、大环化合物构建以及超分子化学等众多领域. 由于篇幅有限，在此我们仅摘录炔偶联反应的应用个例 展示此类化学反应在这些方面的应用.

\section{1 合成非环状炔化合物}

Mohr 等 ${ }^{[65]}$ 应用 Hay 偶联反应合成了一系列链状炔 聚合物(Scheme 19). 他以端炔 37 为起始物, 分别通过 条件 $\mathrm{a}$ 和 $\mathrm{b}$ 得到链状炔化物 38 和炔硅化物 $\mathbf{3 9}$, 用 $n-\mathrm{Bu}_{4} \mathrm{NF}$ 处理 39 得端炔 $\mathbf{4 0}$, 然后采用类似的 Hay 偶联 条件得到更长链炔化物 41 和 $\mathbf{4 2}$. 此例中合成应的含金 属长链炔聚物以其独特的性质应用于新材料领域.

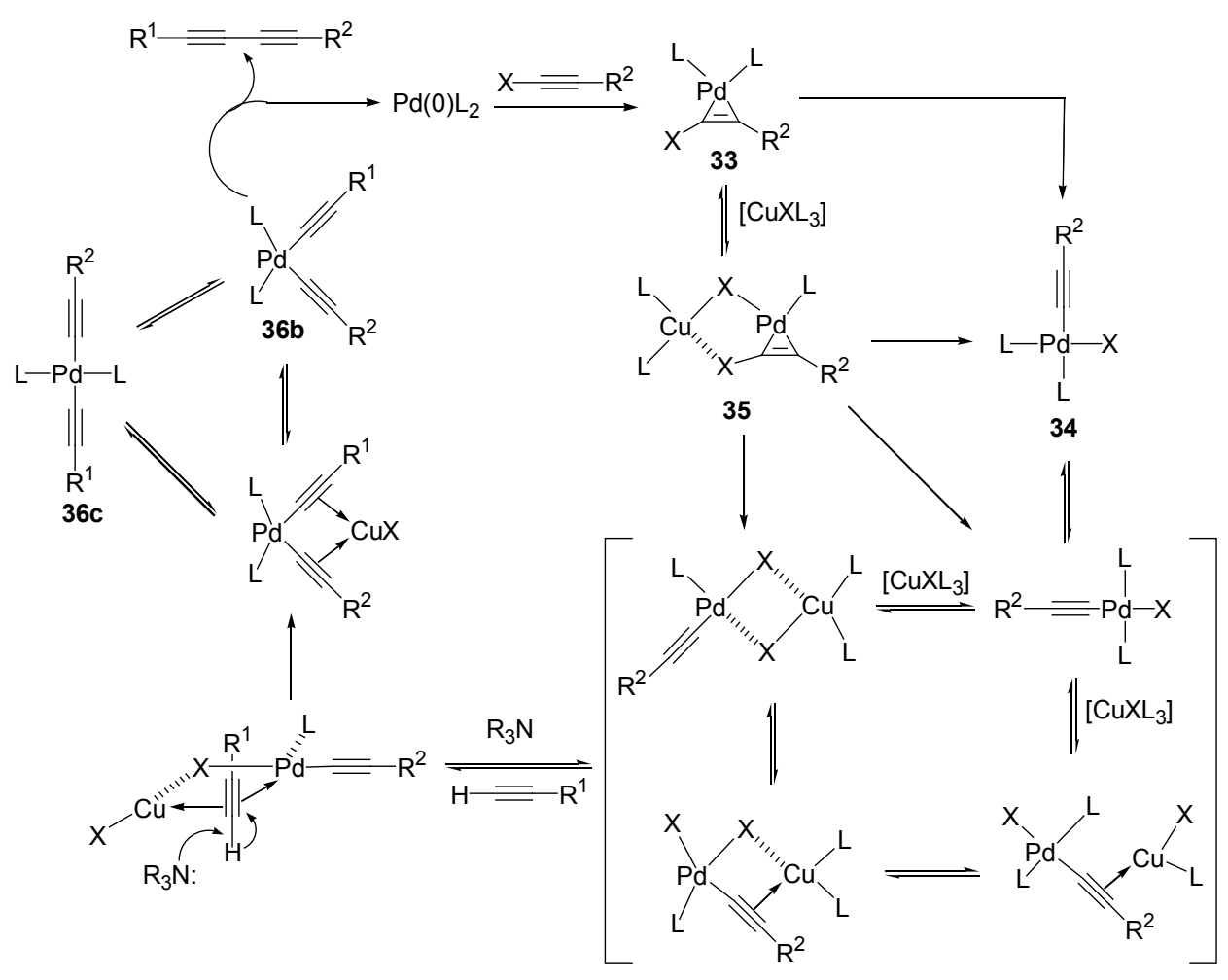

Scheme 17 


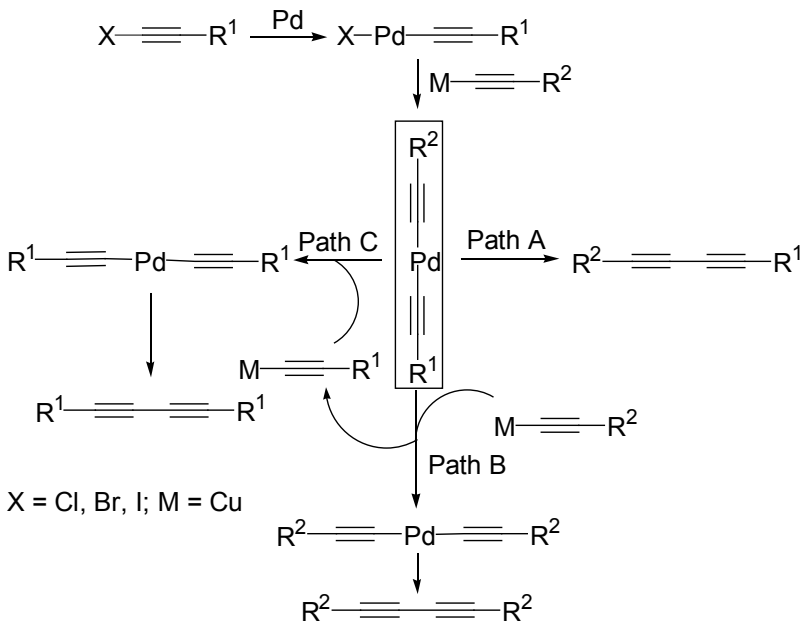

Scheme 18

2009年 Zhao 研究组 ${ }^{[66 a]}$ 把炔偶联反应拓展应用到富 勒烯衍生物的合成中, 他们合成了一系列链状和星状富 勒烯衍生物(图中仅以链状物为例)(Scheme 20). 该合成
中经历 3 个关键反应，一是 $\operatorname{Pd}$ 催化的交叉偶联反应；二 是 $\mathrm{Cu}$ 催化的氧化炔均偶联反应; 三是富勒烯的原位炔 基化反应，通过控制反应条件可以合成形态各异的富勒 烯炔聚物, 后者在纳米材料和光电材料等领域具有潜在 的应用价值.

2011 年，该小组 ${ }^{[66 b]}$ 又将这类一价 $\mathrm{Cu}$ 催化的炔偶联 反应拓展应用到星状大分子炔化物的合成中 (Scheme 21). 这又为合成大分子炔化物提供了方法依据.

此外, 2009 年 Lee 小组 ${ }^{[67]}$ 报道了一例 Pd 催化的树 形炔化物的合成(Eq. 49). 此例中在温和的条件下实现 了大分子端炔之间的高收率均偶联反应.

\section{2 大环系炔化物的构建}

大环系炔化物的合成一直是炔偶联反应的主要应 用之一 ${ }^{[4 b]}$. Tykwinski 等 ${ }^{[68]}$ 报道了应用 $\mathrm{Cu}$ 催化的炔偶联 反应合成大环炔化物(Scheme 22). 43 和 44 经过 Pd 催化 的交叉偶联反应得到单体 $\mathbf{4 5}$ ，单体 $\mathbf{4 5}$ 经条件 $\mathrm{b}$ 脱硅基 然后在一价铜催化下偶联给出环状炔化物 $\mathbf{4 6}$.

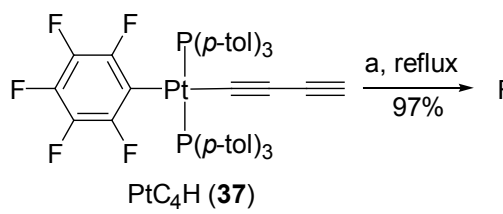

$$
\mid \begin{aligned}
& \mathrm{b}, \text { eflux } \\
& 53 \%
\end{aligned}
$$

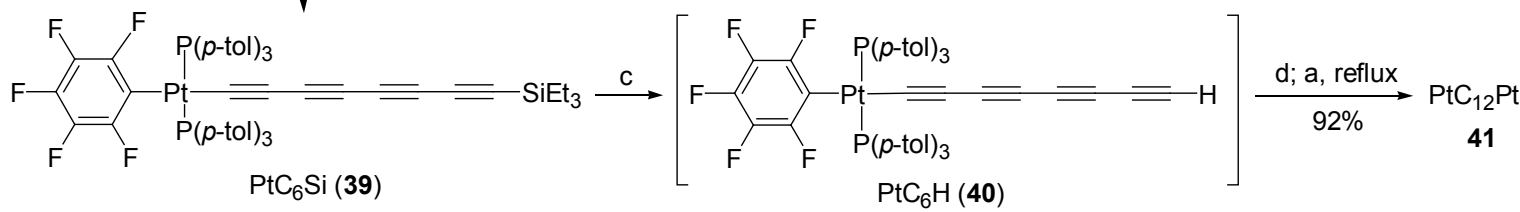

$\mathrm{PtC}_{6} \mathrm{Si}(39)$

$$
\begin{aligned}
& \text { a) } \mathrm{O}_{2} \text {, cat. CuCl/TMEDA, acetone; } \\
& \text { b) } \equiv \mathrm{SiEt}_{3} \text { (excess), } \\
& \mathrm{O}_{2} \text {, cat. CuCl/TMEDA, acetone; } \\
& \text { c) wet } n-\mathrm{Bu}_{4} \mathrm{NF} \text {; } \\
& \text { d) } \mathrm{ClSiMe}_{3}
\end{aligned}
$$

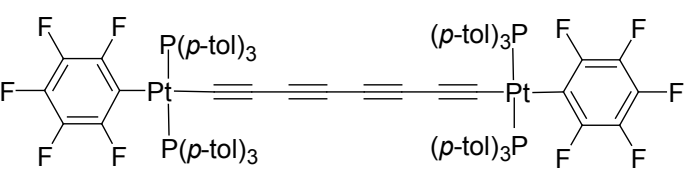

$\mathrm{PtC}_{8} \mathrm{Pt}(38)$

$\mathrm{PtC}_{6} \mathrm{H}(\mathbf{4 0})$

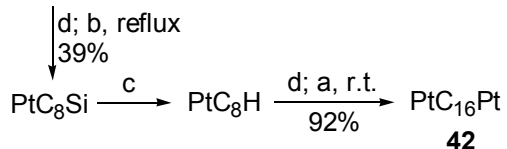

Scheme 19
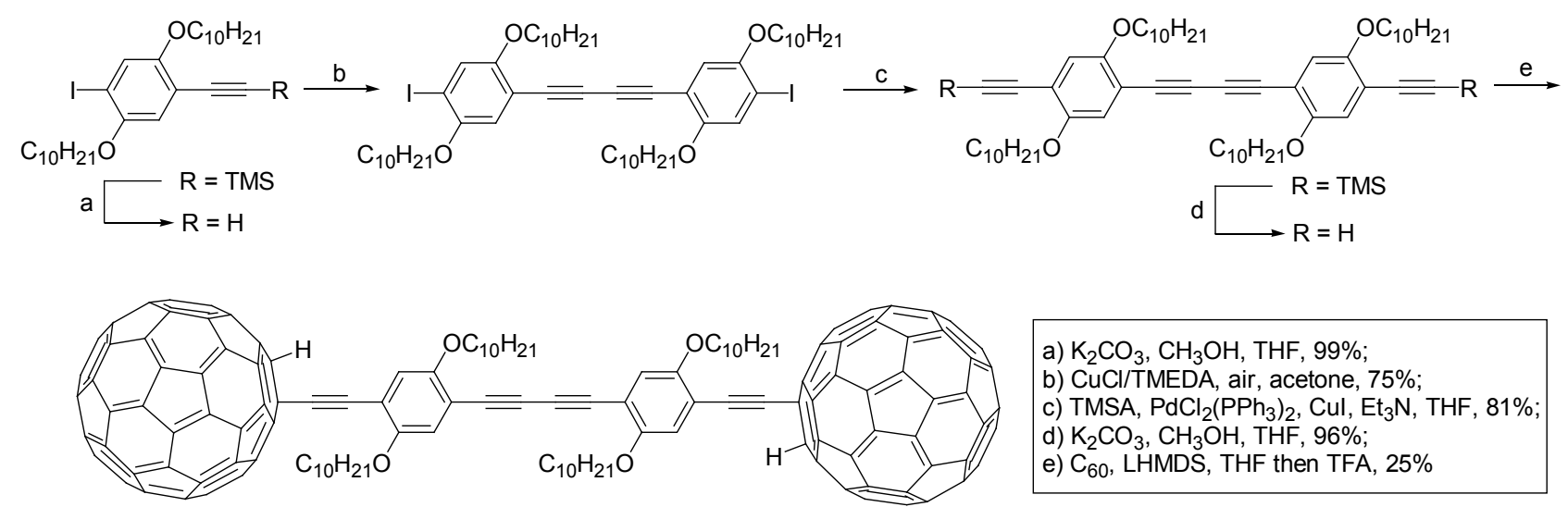

a) $\mathrm{K}_{2} \mathrm{CO}_{3}, \mathrm{CH}_{3} \mathrm{OH}, \mathrm{THF}, 99 \%$;

b) CuCI/TMEDA, air, acetone, $75 \%$;

c) TMSA, $\mathrm{PdCl}_{2}\left(\mathrm{PPh}_{3}\right)_{2}, \mathrm{Cul}, \mathrm{Et}_{3} \mathrm{~N}, \mathrm{THF}, 81 \%$;

d) $\mathrm{K}_{2} \mathrm{CO}_{3}, \mathrm{CH}_{3} \mathrm{OH}$, THF, $96 \%$;

e) $\mathrm{C}_{60}$, LHMDS, THF then TFA, $25 \%$

Scheme 20 

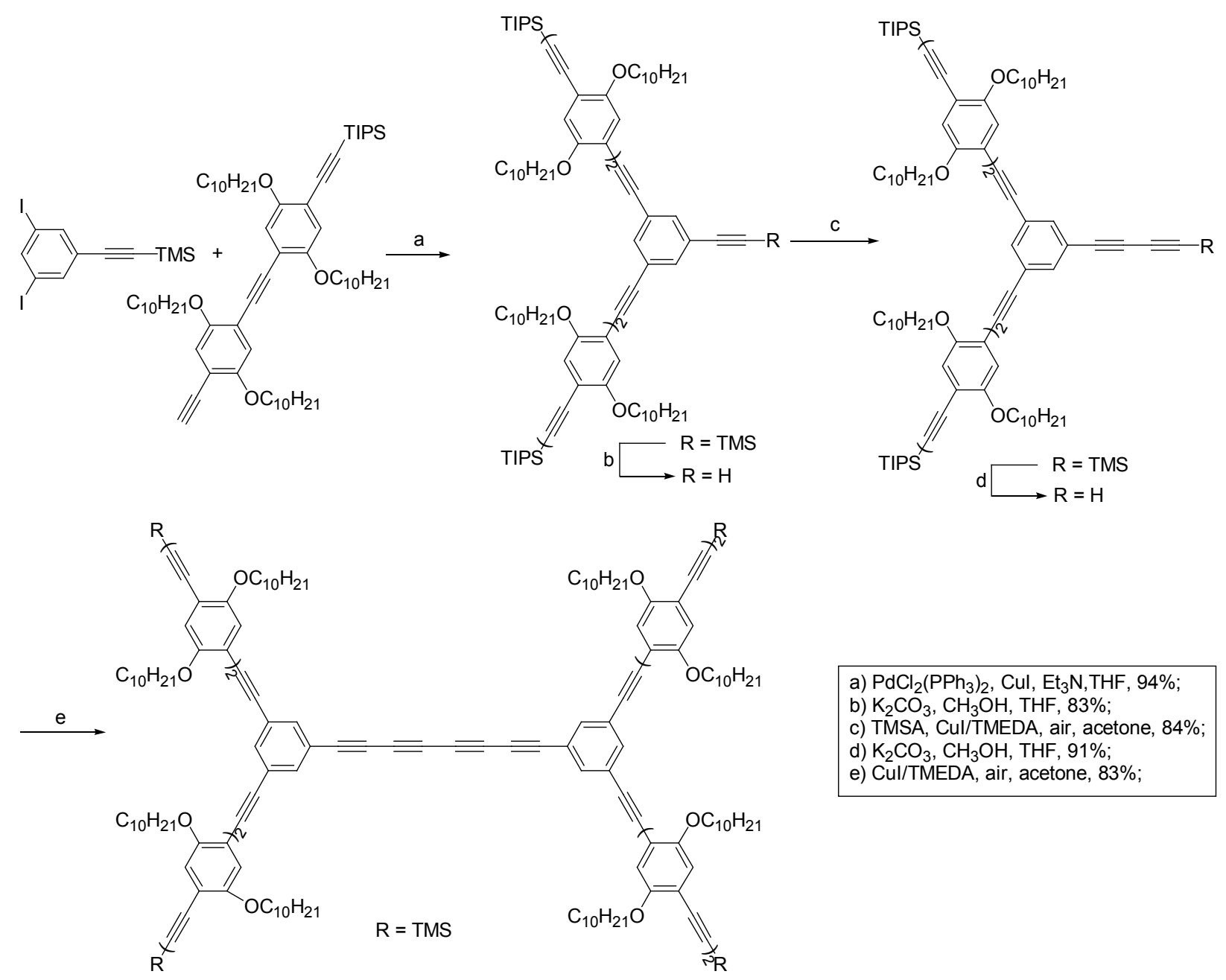

a) $\mathrm{PdCl}_{2}\left(\mathrm{PPh}_{3}\right)_{2}$, Cul, $\mathrm{Et}_{3} \mathrm{~N}, \mathrm{THF}, 94 \%$;

b) $\mathrm{K}_{2} \mathrm{CO}_{3}, \mathrm{CH}_{3} \mathrm{OH}, \mathrm{THF}, 83 \%$;

c) TMSA, Cul/TMEDA, air, acetone, $84 \%$;

d) $\mathrm{K}_{2} \mathrm{CO}_{3}, \mathrm{CH}_{3} \mathrm{OH}$, THF, $91 \%$;

e) Cul/TMEDA, air, acetone, $83 \%$;

Scheme 21
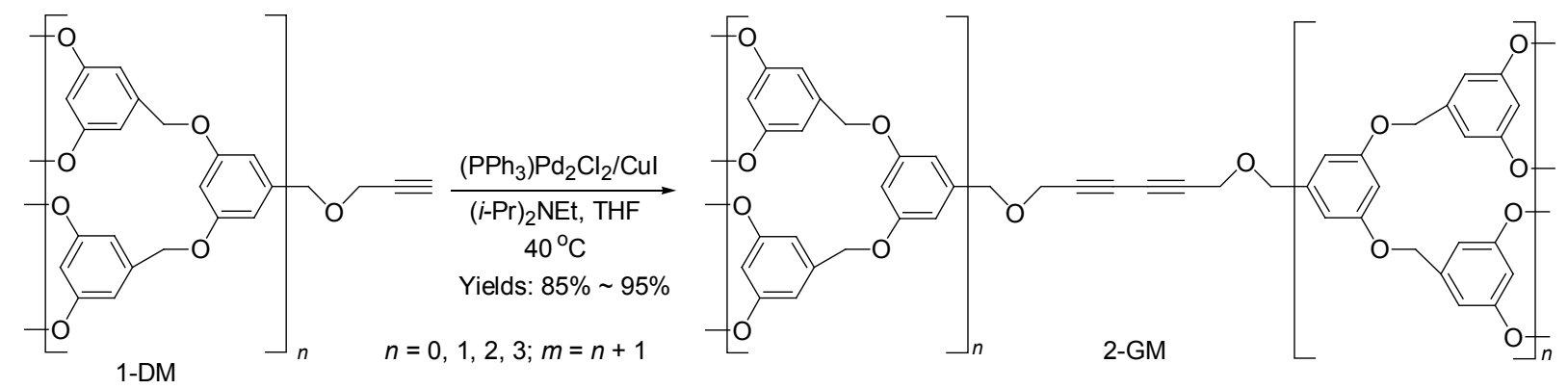

Haloy 等 ${ }^{[69]}$ 通过炔偶联合成了一系列具有复杂结构 的炔化物这类大环化合物在以前的综述文章中有过报 道, 这里不再赘述.

2011 年, Zhao 小组 ${ }^{[66 b]}$ 也报道了通过 Pd 催化的炔偶 联构建大环炔化物的例子(Scheme 23). 此例中同样采 用硅基保护端炔的方法, 最后通过脱硅基, Pd 催化的炔 偶联反应给出大环炔化物.

\section{3 天然产物合成中的应用}

由于炔属结构广泛存在于天然产物中, 所以炔偶联
反应在天然产物的合成中也有广泛应用. 这部分工作在 2006 年的综述中有详尽总结 ${ }^{[2]} .2011$ 年, Swain 等 ${ }^{[70]}$ 又将 炔偶联方法应用到 panaxytriol 和 panaxydiol 的合成中 (Scheme 24), 其中 Panaxytriol 是红参中分离出的具有强 抗肿瘤活性的物质. 该路线中用到了 Cadiot-Chodkiewicz 端炔杂偶联反应.

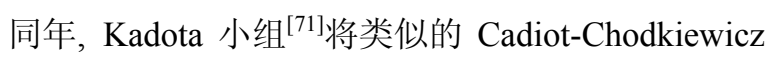
端炔交叉偶联反应应用到(一)-gummiferol 的合成中 (Scheme 25). 


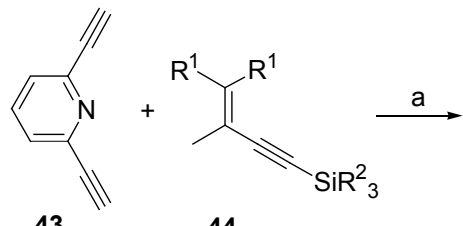

43

44

a) $\mathrm{Pd}\left(\mathrm{PPh}_{3}\right)_{4}$, Cul, $\mathrm{Et}_{2} \mathrm{NH}$, THF,r.t.; b) $\mathrm{NaOH}$, THF/MeOH, r.t.;

c) Cul, TMEDA, $\mathrm{CH}_{2} \mathrm{Cl}_{2}, \mathrm{O}_{2}$, r.t.

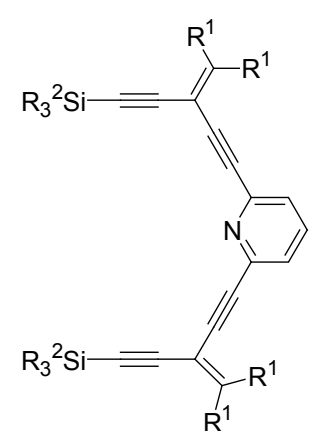

45a $R^{1}=M e, R^{2}=$ Me $81 \%$ 45b $R^{1}=P h, R^{2}=$ Et $49 \%$

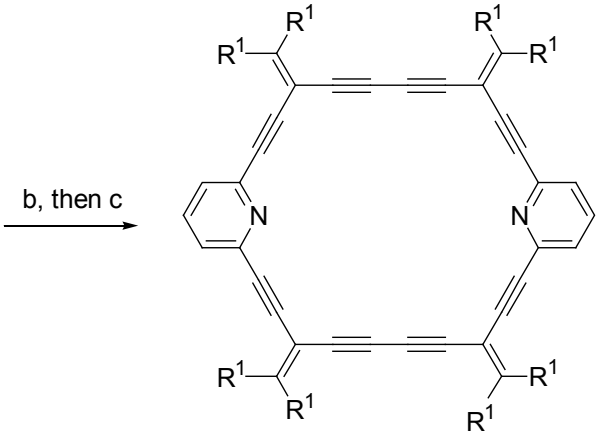

46a $\mathrm{R}^{1}=$ Me not isolated 46b $\mathrm{R}^{1}=\mathrm{Ph} 62 \%$

Scheme 22
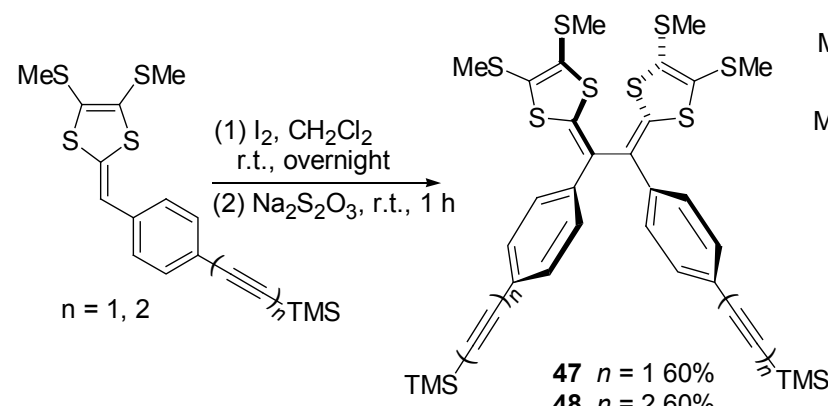

$$
\begin{aligned}
& 47 \underset{\text { (1) } \mathrm{K}_{2} \mathrm{CO}_{3}, \mathrm{THF} / \mathrm{MeOH}}{\stackrel{(2) \mathrm{PdCl}_{2}\left(\mathrm{PPh}_{3}\right)_{2}, \mathrm{Cul}, \mathrm{TMEDA}}{\text { acetone, air, reflux, } 48 \mathrm{~h}, 18 \%}} \mathbf{4 8} \\
& 48 \underset{\text { (1) } \mathrm{K}_{2} \mathrm{CO}_{3}, \mathrm{THF} / \mathrm{MeOH}}{\stackrel{(2) \mathrm{PdCl}_{2}\left(\mathrm{PPh}_{3}\right)_{2}, \mathrm{Cul}, \mathrm{TMEDA}}{\text { acetone, air, reflux, 120 h }}} \mathbf{5 0}
\end{aligned}
$$

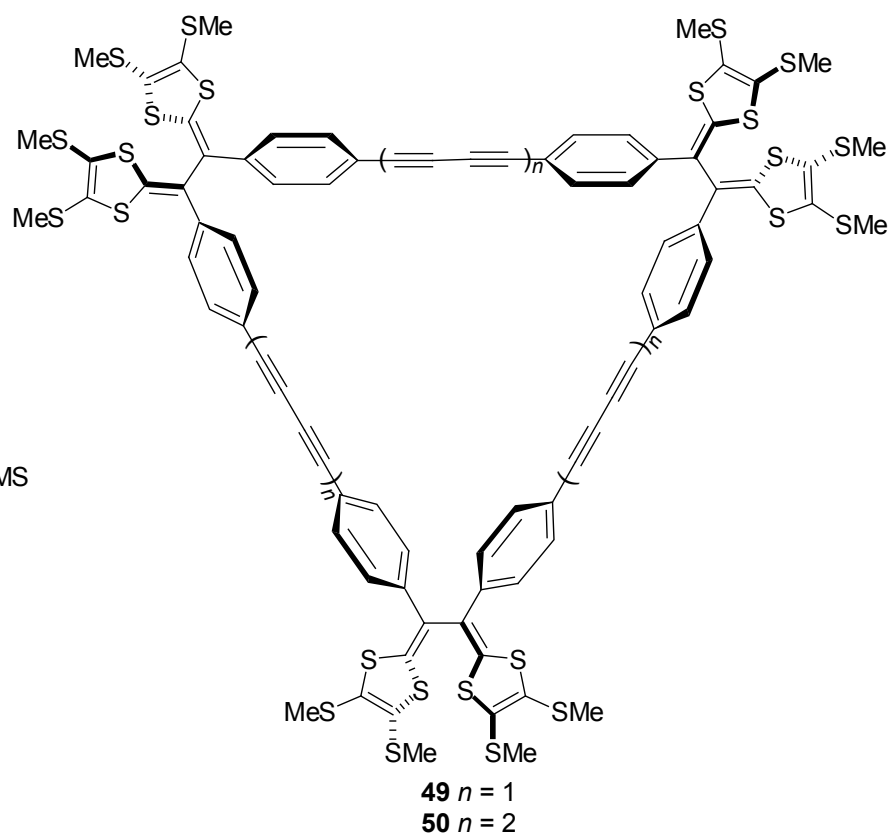

Scheme 23

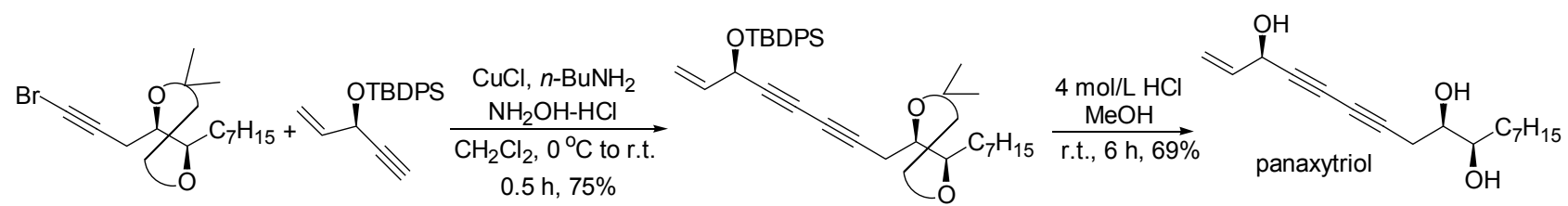

Scheme 24

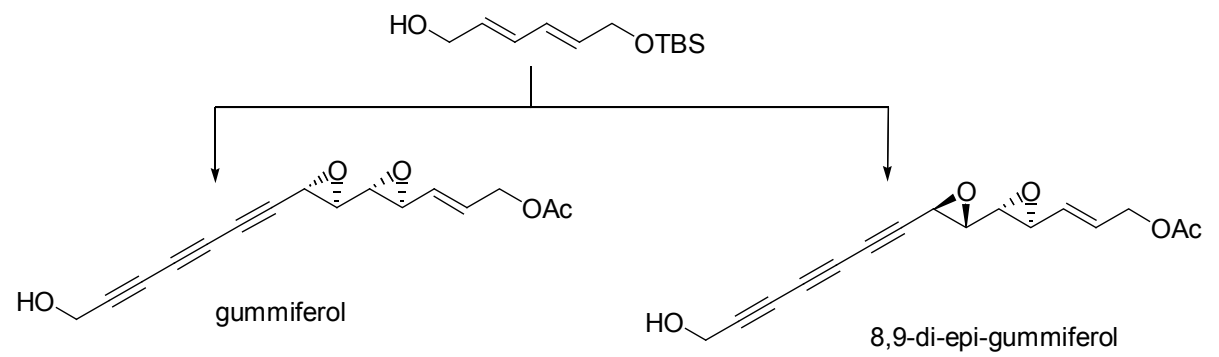

Scheme 25 


\section{4 超分子领域的应用}

炔偶联反应是一类有效合成轮烯的方法，而后者在 分子器件、材料和分子机器领域有潜在的应用价值. 近 几年这方便的报道也不在少数 ${ }^{[72 \mathrm{a}, 72 \mathrm{~b}, 72 \mathrm{c}]}$, 这里以个例说 明.

2006年, Saito 等 ${ }^{[73]}$ 就报道了一例通过 Glaser 偶联反 应合成轮烷的例子(Eq. 50). 端炔 $\mathbf{5 1}$ 与等量的邻二氮菲 衍生物 $\mathbf{5 2}$ 通过 Galser 偶联反应以 $72 \%$ 的收率得到轮烷 53.

2011 年, Langton 等 ${ }^{[74]}$ 也报道了类似的铜催化炔偶 联反应应用于该领域的实例(Eq. 51). 该反应应用一价 铜盐 $\mathrm{CuI}$ 促进大分子端炔的偶联, 从而实现分子识别.
当然，除了以上几个方面的应用外，炔偶联反应在 高分子化学、功能材料、发光材料以及表面化学等方面 也有应用 ${ }^{[46]}$, 这里就不一一展开说明.

综上所述, 近些年涌现出很多条件温和, 操作方便 的炔偶联方法, 尤其是铜试剂和钯试剂催化的炔偶联方 法. 这些方法极大的丰富和发展了此类化学反应. 但由 于近年来涉及机理方面的研究较少, 故未能给出指导此 类反应改进的有效理论支撑, 相信在以后的研究中这方 面会有所突破. 此外，近年来许多涉及炔偶联反应应用 的文献报道格外引人注目. 由于炔偶联反应的诸多应用 必将促使更多的研究者继续深入这方面的研究工作. 相 信以后会取得更加丰硕的研究成果.

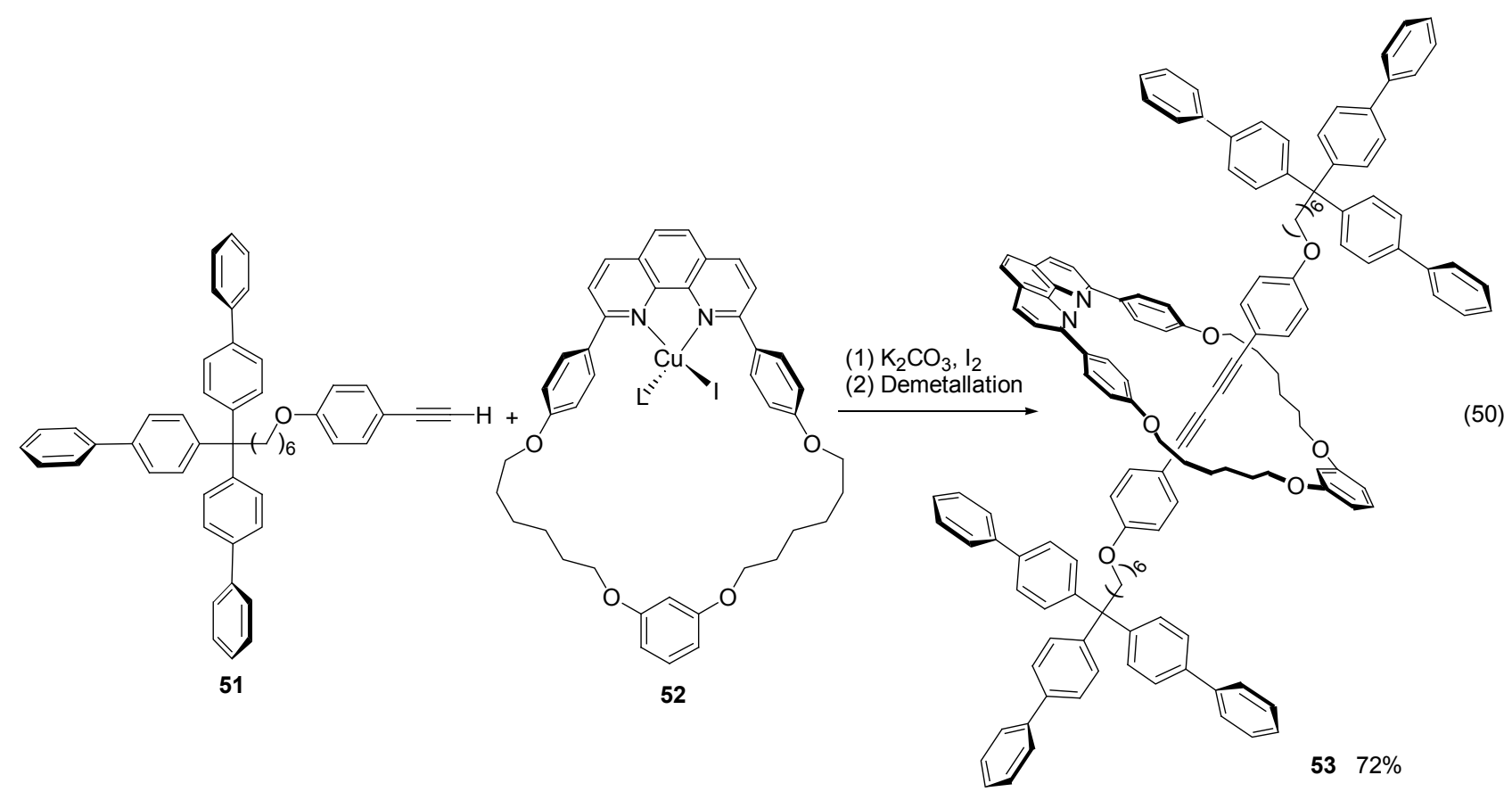

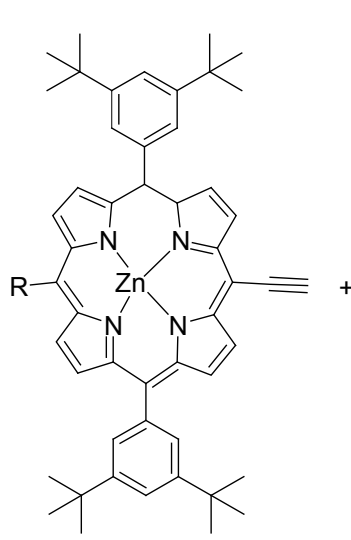

$\mathrm{R}=\mathrm{Ph}, \mathrm{R}=\mathrm{C}_{2} \mathrm{Si}\left(i-\mathrm{Pr}_{3}\right)$

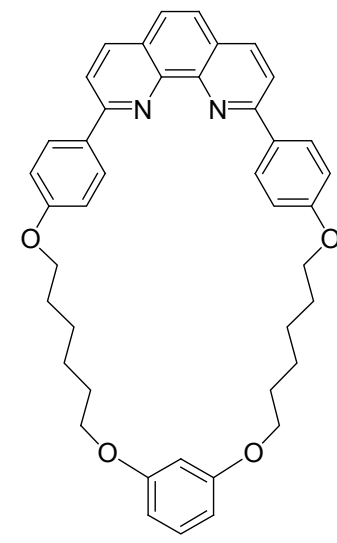

$\mathrm{M}$

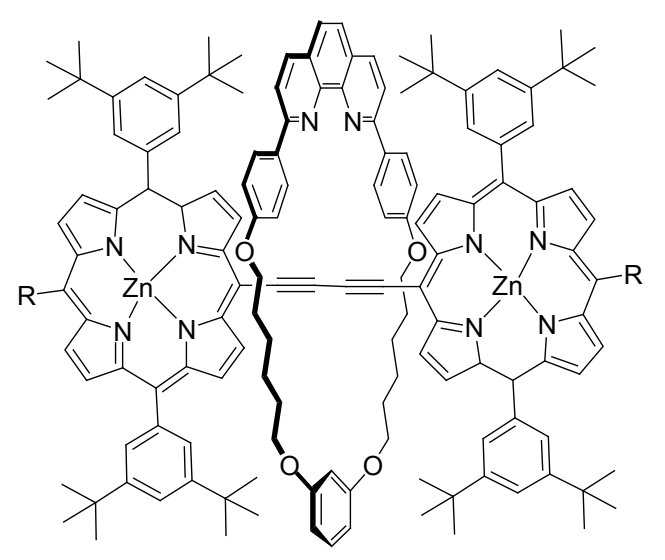

$\mathrm{R}=\mathrm{Ph}, 42 \%$

TBAF 


\section{References}

[1] (a) Glaser, C. Ber. Dtsch. Chem. Ges. 1869, 2, 422. (b) Glaser, C. Ann. Chem. Pharm. 1870, 154, 137.

[2] Annabelle, L. K.; Shun, S.; Tykwinski, R. Angew. Chem. Int. Ed. 2006, 45,1034

[3] Xu, Z. F.; Kaha, M.; Walker, K. L. J. Am. Chem. Soc. 1994, 116, 4537.

[4] (a) Sienmsen, P.; Livingston, R. C.; Diederich,F. Angew. Chem. Int. Ed. 2000, 39, 2632.

(b) Stefani, H. A.; Guarezemini, A. S.; Cella, R. Tetrahedron 2010 , 66, 7871 .

[5] Tang, J. Y.; Jiang, H. F.; Deng, G. H.; Zhou, L. Chin. J. Org Chem. 2005, 25, 1503 (in Chinese). (唐金玉，江焕峰，邓国华，周砧，有机化学, 2005, 25, 1503.)

[6] Eglinton, G.; Galbraith, A. R. Chem. Ind. (London) 1956, 737.

[7] Kürti, L.; Czakó, B. Strategic Applications of Named Reactions in Organic Synthesis, Science Press, Beijing, 2007, p. 186.

[8] Hay, A. S. J. Org. Chem. 1962, 27, 3320

[9] Yadav, J. S.; Reddy, B. V. S.; Bhaskar Reddy, K.; Gayathri, U. K.; Prasad, A. R. Tetrahedron Lett. 2003, 44, 6493.

[10] Ranu, B. C.; Banerjee, S. Lett. Org. Chem. 2006, 3, 607.

[11] Ye, C.; Xiao, J.; Twamley, B.; Shreeve, J. M. Eur. J. Org. Chem. 2007, 5095 .

[12] (a) Li, J.; Jiang, H. Chem. Commun. 1999, 2369.

(b) Jiang, H.; Tang, J.; Wang, A.; Deng, G.; Yang, S. Synthesis 2006, 1155 .

[13] (a) Kuhn, P.; Sommer, J. Eur. J. Org. Chem. 2009, 423.

(b) Kuhn, P.; Pale, P.; Sommer, J.; Louis, B. J. Phys. Chem. C 2009, 113, 2903.

[14] Alix, A.; Pale, P. Synthesis 2010, 1557.

[15] Wang, D.; Li, J.; Gao, T.; Hou, S.; Chen, B. Green Chem. 2010, 12, 45.

[16] (a) Yin, K.; Li, C.; Li, J.; Jia, X. Green Chem. 2011, 13, 591. (b) Yin, K.; Li, C.; Li, J.; Jia, X. Appl. Organomet. Chem. 2011, 25, 16.

(c) Li, D.; Yin, K.; Li, J.; Jia, X. Tetrahedron Lett. 2008, 49, 5918.

(d) Jia, X.; Yin, K.; Li, C.; Li, J.; Bian, H. Green Chem. 2011, 13, 2175 .

[17] Schmidt, R.; Thorwirth, R.; Szuppa, T.; Stolle, A.; Ondruschka, B.; Hopf, H. Chem. Eur. J. 2011, 17, 8129.

[18] Oishi, T.; Yamaguchi, K.; Mizuno, N. ACS Catal. 2011, 1, 1351.

[19] Wu, T.; Huang, S.; Tsai, F. Appl. Organomet. Chem. 2011, 25, 395.

[20] Zhang, S.; Liu, X.; Wang, T. Adv. Synth. Catal. 2011, 353, 1463.

[21] Zhu, B. C.; Jiang, X. Z. Appl. Organomet. Chem. 2007, 21, 345.

[22] Nador, F.; Fortunato, L.; Moglie, Y.; Vitale, C.; Radivoy, G. Synthesis 2009, 4027.

[23] Alonso, F.; Melkonian, T. Moglie, Y.; Yus, M. Eur. J. Org. Chem. 2011, 2524

[24] Oishi, T.; Katayama, T.; Yamaguchi, K.; Mizuno, N. Chem. Eur. J. 2009, 15, 7539.

[25] Balamurugan, R.; Naveen, N.; Manojveer, S.; Nama, M. V. Aust. J. Chem. 2011, 64, 567.

[26] Balaraman, K.; Kesavan, V. Synthesis 2010, 3461.

[27] Kusuda, A.; Xu, X. H.; Wang, X.; Tokunaga, E.; Shibata, N. Green Chem. 2011, 13,843.

[28] Rossi, R.; Carpita, A.; Bigelli, C. Tetrahedron Lett. 1985, 26, 523.

[29] (a) Liu, Q.; Burton, D. J. Tetrahedron Lett. 1997, 38, 4371. (b) Hattori, T.; Kijima, M.; Shirakawa, H. Synth. Met. 1997, 84, 357.

[30] Alonso, D. A.; Najera, C.; Pacheco, M. Adv. Synth. Catal. 2003, $345,1146$.
[31] Gil-Moltó, J.; Najerá, C. Eur. J. Org. Chem. 2005, 4073.

[32] Zhou, L.; Zhan, H.-Y.; Liu, H.-L.; Jiang, H.-F. Chin. J. Chem. 2007, $25,1413$.

[33] Kurita, T.; Abe, M.; Maegawa, T.; Monguchi, Y.; Sajiki, H. Synlett 2007, 2521.

[34] Watarai, N.; Kawasaki, H.; Saito, S. Heterocycles 2009, 79, 531.

[35] Perrone, S.; Bona, F.; Troisi, L. Tetrahedron 2011, 67, 7386.

[36] Li, J. X.; Liang, H. R.; Wang, Z. Y.; Fu, J. H. Monatsh. Chem. 2011, 142, 507.

[37] Bharathi, P.; Periasamy, M. Organometallics 2000, 19, 5511.

[38] Krafft, M. E.; Hirosawa, C.; Dalal, N.; Ramsey, C.; Stiegman, A. Tetrahedron Lett. 2001, 42, 7733.

[39] Hilt, G.; Hengst, C.; Arndt, M. Synthesis 2009, 395.

[40] Yin, W.; He, C.; Chen, M.; Zhang, H.; Lei, A. Org. Lett. 2009, 11, 709.

[41] Peng, J.; Liu, X.; Kishi, Y. Tetrahedron Lett. 2011, 52, 2172.

[42] (a) Yamaguchi, K.; Kamata, K.; Mizuno, N. J. Catal. 2008, 258, 121.

(b) Mizuno, N.; Yamaguchi, K.; Kamata, K. Catal. Surv. Asia 2011, 15, 68 .

[43] (a) Chodkiewicz, W.; Cadiot, P.; Hebd, C. R. Hebd. Seance Acad. Sci. 1955, 241, 1055 .

(b) Chodkiewicz, W. Ann. Chim. (Paris) 1957, 2, 819

[44] Shen, W.; Thomas, S. A. Org. Lett. 2000, 2, 2857.

[45] Shi, W.; Luo, Y.; Luo, X.; Lei, A. J. Am. Chem. Soc. 2008, 130, 14713.

[46] Damle, S.; Seomoon, D.; Lee, P. H. J. Org. Chem. 2003, 68, 7085.

[47] Xue, S.; Meng, L.; Guo, Q. Synth. Commun. 2008, 38, 2243.

[48] Chen, Z.; Jiang, H.; Wang, A.; Yang, S. J. Org. Chem. 2010, 75, 6700 .

[49] Cahiez, G.; Duplais, C.; Buendia, J. Angew. Chem., Int. Ed. 2009 48,6731 .

[50] Kude, K.; Hayase, S.; Kawatsura, M. Itoh, T. Heteroat. Chem. 2011, 22, 398

[51] Nishihara, Y.; Ikegashira, K.; Mori, A.; Hiyama, T. J. Org. Chem. 2000, 65, 1780.

[52] Yoshida, H.; Yamaryo, Y.; Ohshita, J.; Kunai, A. Chem. Commun 2003, 1510

[53] Oh, C. H.; Reddy, V. R. Tetrahedron Lett. 2004, 45, 5221.

[54] Nishihara, Y.; Okamoto, M.; Inoue, Y. Tetrahedron Lett. 2005, 46, 8661.

[55] Shirakawa, E.; Nakao, Y.; Murota, Y.; Hiyama, T. J. Organomet. Chem. 2003, 670, 132.

[56] Ochiai, M.; Nishi, Y.; Goto, S.; Frohn, H. J. Angew. Chem., Int. Ed. 2005, 44, 406.

[57] Singh, F. V.; Amaral, M.; Stefani, H. A. Tetrahedron Lett. 2009, $50,2636$.

[58] (a) Zalkind, Y. S.; Fundyler, F. B. Ber. Chem. Ges. 1936, 69, 128. (b) Zalkind, Y. S.; Fundyler, F. B. J. Gen. Chem. USSR 1939, 9, 1725 .

[59] Clifford, A. A.; Water, W. A. J. Chem. Soc. 1963, 3056.

[60] Bohlmann, F.; Schonowsky, H.; Inhoffen, E.; Grau, G. Chem. Ber. 1964, 97, 794.

[61] Alper, H.; Maldonado, M. Organometallics 1989, 8, 1124.

[62] Liu, Q.; Burton, D. J. Tetrahedron Lett. 1997, 38, 4371.

[63] Bumagin, N. A.; Ponomarev, A. B.; Beletskaya, I. P. Bull. Acad. Sci. USSR 1984, 33, 1433.

[64] Cai, C.; Vasella, A. Helv. Chim. Acta 1995, 78, 2053.

[65] Mohr, W.; Stahl, J.; Hampel, F.; Gladysz, J. A. Chem. Eur. J. 2003, 9, 3324

[66] (a) Zhou, N.; Zhao, Y. J. Org. Chem. 2010, 75, 1498.

(b) Chen, G.; Mahmud,I.; Dawe, L. N.; Zhao, Y. J. Org. Chem. 
2011, 76, 2701.

[67] Lee, J. W.; Han, S. C.; Lee, U.; Kim, B.; Kim, J. H. Bull. Korean Chem. Soc. 2009, 30, 1925.

[68] Campbell, K.; Tiemstra, N. M.; Tykwinski, R. Synlett 2004, 182.

[69] Takeda, T.; Fix, A. G.; Haley, M. Org. Lett. 2010, 12, 3824.

[70] Prasad, K. R.; Swain, B. Tetrahedron: Asymmetry 2011, 22, 1261.

[71] Takamura, H.; Wada, H.; Lu, N.; Kadota, I. Org. Lett. 2011, 13, 3644.

[72] (a) Berna, J.; Goldup, S. M.; Lee, A. L.; Leigh, D. A.; Zerbetto, F.
Angew. Chem., Int. Ed. 2008, 47, 4392.

(b) Goldup, S. M.; Leigh, D. A.; Long, T.; McGonigal, P. R.; Symes, M. D.; Wu, J. J. Am. Chem. Soc. 2009, 131, 15924.

(c) Crowley, J. D.; Goldup, S. M.; Gowans, N. D.; Leigh, D. A. J. Am. Chem. Soc. 2010, 132, 6243.

[73] Saito, S.; Takahashi, E.; Nakazono, K. Org. Lett. 2006, 8, 5133.

[74] Langton, M. J.; Matichak, J. D.; Thompson, A. L.; Anderson, H. L. Chem. Sci. 2011, 2, 1897. 